\title{
Free-carrier-driven Kerr frequency comb in optical microcavities: Steady state, bistability, self-pulsation, and modulation instability
}

\author{
R. Haldar, ${ }^{1, *}$ A. Roy, ${ }^{1,2}$ P. Mondal, ${ }^{3}$ V. Mishra, ${ }^{3}$ and S. K. Varshney ${ }^{1}$ \\ ${ }^{1}$ Department of Electronics and Electrical Communication Engineering, IIT Kharagpur-721302, West Bengal, India \\ ${ }^{2}$ Electrical Engineering Department, California Institute of Technology, 1200 East California Boulevard, Pasadena, California 91125, USA \\ ${ }^{3}$ Department of Physics, IIT Kharagpur-721302, West Bengal, India
}

(Received 16 January 2019; published 25 March 2019)

\begin{abstract}
Continuous-wave pumped optical microresonators have been vastly exploited to generate a frequency comb (FC) utilizing the Kerr nonlinearity. Most of the nonlinear materials used to build photonic platforms exhibit nonlinear losses such as multiphoton absorption, free-carrier absorption, and free-carrier dispersion which can strongly affect their nonlinear performances. In this work, we model the Kerr FC based on a modified Lugiato-Lefever equation (LLE) along with the rate equation and develop analytical formulations to make a quick estimation of the steady state, bistability, self-pulsation, and modulation instability (MI) gain and bandwidth in the presence of nonlinear losses. The analytical model is valid over a broad wavelength range as it includes the effects of all nonlinear losses. Higher-order $(>3)$ characteristic polynomials of intracavity power describing the steady-state homogeneous solution of the modified LLE are discussed in detail. We derive the generalized analytical expressions for the threshold (normalized) pump detuning that initiates the optical bistability when nonlinear losses are present. Free-carrier dispersion-led nonlinear cavity detuning is observed through the reverse Kerr tilt of the resonant peaks. We further deduce the expressions of the threshold pump intensity and the range of possible cavity detuning for the initiation of the MI considering the presence of nonlinear losses. The proposed model will be helpful in explaining several numerical and experimental results which have been previously reported and thereby will be able to provide a better understanding of the comb dynamics.
\end{abstract}

DOI: 10.1103/PhysRevA.99.033848

\section{INTRODUCTION}

The optical frequency comb (FC) is a set of equidistant and coherent frequency lines in the ultraviolet, visible, and infrared regions [1], which can be used in precision measurement [2]; microwave signal synthesis [3]; optical communication $[4,5]$; sensing, spectroscopy, and molecular fingerprinting $[2,4,6]$; astronomy [7]; entangled photon pair generations [8]; or as an optical ruler [1,4,9]. Mode-locked femtosecond lasers and fiber lasers have extensively been used for the generation of optical FC [10,11]; until recently the parametric frequency conversion using a continuous wave $(\mathrm{CW})$ optical pump in optical microcavities (microspheres, microdisks, and microring resonators) has revolutionized the technology for comb generation $[12,13]$. Eventually, the device footprint has been reduced to a few hundred micrometers while a repetition rate as high as $>10 \mathrm{GHz}$ can easily be achieved $[12,13]$. Varieties of materials have been used along with different novel fabrication techniques to design ultrahigh-quality-factor microresonators $[4,14,15]$ for low-threshold, stable FC generation; however, the search for the most suitable material is still on. FC generation in crystalline fluorides [16-19], Hydex glass [20], diamond [21], quartz [22], aluminum nitride [23,24], lithium niobate [25], AlGaAs [26], silica [4,12], and silicon nitride [27] have already been demonstrated. Apart from this, FC is also demonstrated in organically modified

\footnotetext{
*raktim@ece.iitkgp.ernet.in
}

silica microcavities by Shen et al. in 2017 [28] with a very low threshold power.

Silicon-based platforms are often preferred due to several advantages such as tight optical confinement, high Kerr coefficient, transparency over a broad wavelength range (telecom to mid-IR), low cost, and most importantly its compatibility with the existing microelectronics industry [29]. High-refractiveindex (RI) contrast between silicon and other cladding materials (air, silica) results in strong optical confinement in silicon waveguides which allows sharp waveguide bends that help to reduce the device footprint [30]. Tight confinement also enhances the effective nonlinearity which facilitates the realization of different nonlinear phenomena with a very low input power [31,32]. It has been shown that efficient dispersion engineering in slot waveguides has the potential to achieve broadband frequency combs [33]. Silicon, on the other hand, exhibits strong two-photon absorption (2PA) when operating below a $2.2-\mu \mathrm{m}$ wavelength $[34,35]$, which has been exploited to realize all-optical logic operations [36] as well as all-optical signal processing [37]. Note that nonlinear RI, 2PA, free-carrier absorption, etc., depend on the material structure, band-gap energy, and wavelength of operations. The allotropes of silicon, viz., crystalline silicon $(c-\mathrm{Si})$, and amorphous silicon $(a-\mathrm{Si})$ having band-gap energies of 1.1 and $\sim 1.6-1.7 \mathrm{eV}$, respectively, are being used in various optical applications [38]. Although optical waveguides made of $c$-Si have a very low linear propagation loss $(0.2-1 \mathrm{~dB} / \mathrm{cm})$ and high $\chi^{(3)}$ nonlinearity, high 2PA reduces the figure of merit (FOM) of $c$-Si in nonlinear applications [32], thereby 
restricting the use of $c-\mathrm{Si}$ in most of the nonlinear and quantum photonic applications in the telecom and near-IR (NIR) wavelength ranges [39]. $a$-Si is easier to deposit and therefore has been used to fabricate slot waveguides [40]. The waveguides made of $a$-Si have relatively higher propagation loss $(\sim 6-7 \mathrm{~dB} / \mathrm{cm})$ due to high material absorption [40]. If hydrogenated $(a-\mathrm{Si}: \mathrm{H})$, the effective band (mobility) gap of $a$-Si can increase up to $1.9 \mathrm{eV}$ [38]. Interestingly, though the effective band-gap energy of $a$-Si or $a-\mathrm{Si}: \mathrm{H}$ is much higher than the $c$-Si, still $a$-Si and $a$-Si:H exhibit comparatively larger 2PA in the $C$ band due to the amorphous nature of the material (i.e., the presence of exponential band tails) [38]. However, overall FOM for $a$-Si:H can be improved by five to 15 times as the nonlinear RI of $a-\mathrm{Si}: \mathrm{H}$ is an order higher than that of $c$-Si. [38].

Apart from silicon, GaAs, AlGaAs, Ge, etc., have 2PA in the $C$ band owing to their band-gap energies, though AlGaAs is identified as one of the potential nonlinear materials for FC generation due to its very high nonlinear refractive index [26] and is popularly known as the "silicon of nonlinear optics"! $\mathrm{SiC}$, chalcogenide glasses such as $\mathrm{As}_{2} \mathrm{~S}_{3}, \mathrm{As}_{2} \mathrm{Se}_{3}$ possess three-photon absorption (3PA), whereas $\mathrm{GaN}$ has four-photon absorption (4PA) in telecommunication wavelengths. Silicon also exhibits 3PA and 4PA losses in mid-IR and far-IR wavelength ranges [41]. Due to its low nonlinear losses siliconnitride $\left(\mathrm{Si}_{3} \mathrm{~N}_{4}\right)$ is often a preferred choice over silicon for nonlinear applications [42] at an expense of Kerr coefficient (an order less than the silicon). Recently, an octave-spanning frequency comb has been demonstrated both in $\mathrm{Si}_{3} \mathrm{~N}_{4}$ [43] and Si microring resonators (MRRs) $[44,45]$. Thus, a more realistic theoretical study on comb dynamics applicable for a broad wavelength range of operation becomes indispensable where all the nonlinear losses and higher-order dispersion terms are being considered.

Nonlinear losses include multiphoton absorptions (2PA, 3PA, 4PA), free-carrier absorption (FCA), and free-carrier dispersion (FCD). A generalized mean-field Lugiato-Lefever equation (LLE) can be used to model the Kerr FC in a high- $Q$, high-finesse optical microcavity where the dispersion, nonlinear phase accumulation over a round trip, and pump detuning are low [46]. It is known that a temporal cavity soliton (CS) generates Cherenkov radiation (dispersive wave) in the presence of higher-order dispersions which leads to octave-spanning FC [46]. Recently, numerical studies have shown that FCA-induced FCD causes nonlinear cavity detuning which in turn helps to generate optical FC even in the absence of linear detuning of the CW pump [47]. 2PA in telecom-NIR and 3PA with FCA and FCD in short- and mid-IR wavelength range generally inhibit the parametric oscillation in a silicon waveguide [41]. To combat freecarrier-induced nonlinear losses, external reverse bias has been employed across the $p-i$ - $n$ junction [44] which has been fabricated along the cross section of the $\mathrm{Si}$ waveguide. The external bias minimizes the FCA-FCD effects by sweeping the generated free carriers. This method facilitates broadband (2.1-3.5 $\mu \mathrm{m}$ [44] and 2.4-4.3 $\mu \mathrm{m}$ [48]) FC over the mid-IR wavelengths. Breather solitons have been demonstrated both in $\mathrm{Si}_{3} \mathrm{~N}_{4}$ and $\mathrm{Si}$ waveguides and the effect of 3PA along with the corresponding FCA-FCD is taken into consideration in the theoretical models $[48,49]$. Self-pulsating phenomena in the presence of 2PA are also discussed [50]. However, a detailed theoretical study that includes the effects of all nonlinear losses and free carriers on the generation of Kerr comb is still scarce.

In this work we obtain the steady-state homogeneous solutions of free-carrier-driven generalized mean-field LLE and report the reverse Kerr tilt as a consequence of FCAFCD-induced nonlinear cavity detuning [51]. The characteristic polynomials for the steady-state homogeneous solutions of the LLE possessing all the nonlinear losses are derived having an order greater than 3 instead of the well-known cubic polynomial [52]. In subsequent sections, we discuss the threshold detuning to initiate the bistability in the presence of multiphoton absorption and free-carrier effects, which is a necessary condition to obtain FC. Finally, we generalize the existing formulations [52-54] to study the modulation instability (MI) in the presence of higher-order dispersion terms, 2PA, 3PA, 4PA, FCA, and FCD. Most of the parameters used in simulations are taken from [41]. To validate the analytical model we solve the modified LLE along with the coupled rate equations numerically through the split-step Fourier method. Our theoretical study can explain several experimental results $[41,43,48]$ and thereby provides an indepth understanding of the FC dynamics in the most practical scenarios.

\section{THEORETICAL MODEL}

Microresonator-based Kerr FC can be modeled by meanfield LLE. Solving the LLE is computationally less intensive than other methods [46,55] while the numerical results obtained from LLE match with the experiments reasonably well even for octave-spanning FC.

\section{Normalization of LLE}

The generalized mean-field Lugiato-Lefever equation including multiphoton absorption, FCA, and FCD to model the Kerr comb along with the rate equation can be written as [41]

$$
\begin{aligned}
t_{\mathbf{R}} \frac{\partial E(t, T)}{\partial t}= & -\left(\alpha+i \delta_{\mathbf{0}}\right) E(t, T)+i L \sum_{k \geqslant 2} \frac{\beta_{\mathrm{k}}}{k !}\left(i \frac{\partial}{\partial T}\right)^{k} E(t, T)+\left(1+\frac{i}{\omega_{0}} \frac{\partial}{\partial T}\right) \\
& \times\left(i \gamma L|E(t, T)|^{2}-\frac{\beta_{2 \mathbf{P A}} L}{2 A_{\text {eff }}}|E(t, T)|^{2}-\frac{\beta_{3 \mathbf{P A}} L}{3 A_{\text {eff }}^{2}}|E(t, T)|^{4}\right. \\
& \left.-\frac{\beta_{\mathbf{4 P A}} L}{4 A_{\text {eff }}^{3}}|E(t, T)|^{6}\right) E(t, T)-\frac{\sigma L}{2}\left(1+i \mu_{\mathbf{c}}\right) N_{\mathbf{c}}(t, T) E(t, T)+\sqrt{\theta} E_{\mathbf{i n}},
\end{aligned}
$$




$$
\frac{\partial N_{\mathbf{c}}(t, T)}{\partial T}=\frac{\beta_{2 \mathbf{P A}}}{2 \hbar \omega_{\mathbf{0}}} \frac{|E(t, T)|^{4}}{A_{\text {eff }}^{2}}+\frac{\beta_{\mathbf{3 P A}}}{3 \hbar \omega_{0}} \frac{|E(t, T)|^{6}}{A_{\text {eff }}^{3}}+\frac{\beta_{\mathbf{4 P A}}}{4 \hbar \omega_{\mathbf{0}}} \frac{|E(t, T)|^{8}}{A_{\text {eff }}^{4}}-\frac{N_{\mathbf{c}}(t, T)}{\tau_{\text {eff }}},
$$

where $\tau, T$, and $t_{R}$ represent the fast time, slow time, and the round-trip time, respectively. $t_{R}=\mathrm{FSR}^{-1}$ where FSR is the free-spectral range of the cavity. $E(t, T)$ is the field amplitude whereas $\alpha, \Delta_{0}, L, \beta_{k}, \omega_{0}, E_{\text {in }}$ denote the dimensionless total round-trip loss coefficient, external pump detuning, cavity perimeter length, $k$ th order dispersion coefficient, the angular frequency of the CW pump and input pump amplitude, respectively. $\beta_{\mathrm{nPA}}(n=2,3,4), \sigma, \mu_{c}, N_{c}, A_{\mathrm{eff}}, t_{\mathrm{eff}}$, and $\hbar(=h / 2 \pi)$ are the multiphoton absorption coefficient, FCA, FCD, freecarrier density, effective mode area, carrier lifetime, and reduced Planck's constant, respectively. At first, we neglect the self-steepening term and normalize [46] the LLE and the rate equation including multiphoton absorption (nPA), FCA, and FCD terms in convenient compact series forms, as given by Eqs. (3) and (4), respectively, where $n$ runs from 2 to 4.

$$
\begin{gathered}
\frac{\partial u}{\partial \xi}=-(1+i \Delta) u-\frac{1}{2}(1+i K) \phi_{\mathbf{c}} u-i \frac{s}{2} \frac{\partial^{2} u}{\partial \tau^{2}}+\sum_{k \geqslant 3} \frac{d_{\mathrm{k}}}{k !} \frac{\partial^{k} u}{\partial \tau^{k}} \\
+i|u|^{2} u-\sum_{n=2}^{4} \frac{Q_{\mathrm{n}}}{n}|u|^{2(n-1)} u+S \\
\frac{\partial \phi_{c}}{\partial \tau}=\sum_{n=2}^{4} \theta_{c_{n}}|u|^{2 n}-\frac{\phi_{\mathrm{c}}}{\tau_{\mathrm{c}}}
\end{gathered}
$$

$u(\zeta, \tau), \xi, \quad \Delta, \quad K, \quad \phi_{c}, \quad s, \quad d_{k}, \quad Q_{n} \quad(n=2,3,4), \quad S$, $\theta_{\mathrm{cn}}(n=2,3,4)$, and $\tau_{c}$ are the normalized- intracavity field amplitude, slow time, fast time, pump detuning, FCD coefficient, free-carrier density, second- and higher-order dispersion terms, multiphoton absorption coefficients, pump amplitude, FCA coefficients, and normalized carrier lifetime, respectively, where the normalization factors are given in [56]. One can easily calculate the values of normalized parameters that correspond to the experimentally obtained parameters from Table 2 of Ref. [56]. As the carrier lifetime $\left(t_{\mathrm{eff}}\right)$ is usually greater than the round-trip time $\left(t_{R}\right)$, one should consider the carrier accumulation over successive round trips through the boundary condition, $N_{c}\left(t,-t_{R} / 2\right)=N_{c}\left(t+\Delta t,+t_{R} / 2\right)$. Similarly, $t_{c}$ is also greater than the normalized cavity round-trip time $t_{\mathrm{R}}^{\prime}\left(=t_{\mathrm{R}} / L_{D}\right)$ and $\phi_{\mathrm{c}}\left(\xi,-t^{\prime}{ }_{\mathrm{R}} / 2\right)=\phi_{\mathrm{c}}\left(\xi+\Delta \xi,+t^{\prime}{ }_{\mathrm{R}} / 2\right)$. At the steady state, $\phi_{\mathrm{c}}\left(\xi,-t^{\prime}{ }_{\mathrm{R}} / 2\right)=\phi_{\mathrm{c}}\left(\xi+\Delta \xi,+t^{\prime}{ }_{\mathrm{R}} / 2\right)=\phi_{0}$ where $\phi_{0}$ is the steady-state free-carrier concentration. Equations (3) and (4) can be further simplified by assuming the dominant multiphoton absorption terms only in the operating pump wavelength range. This assumption holds satisfactorily true when the nonlinear losses either inhibit the formation of FC or significantly reduce the span of the frequency comb. Nevertheless, Eqs. (3) and (4) are more suitable if the FC is octave spanned. Therefore, Eqs. (3) and (4) that include only the dominant nPA, FCA, FCD, and rate equations can be rewritten as

$$
\begin{gathered}
\frac{\partial u}{\partial \xi}=-(1+i \Delta) u-\frac{1}{2}(1+i K) \phi_{\mathrm{c}} u-i \frac{s}{2} \frac{\partial^{2} u}{\partial \tau^{2}} \\
+\sum_{k \geqslant 3} \frac{d_{\mathrm{k}}}{k !} \frac{\partial^{k} u}{\partial \tau^{k}}+i|u|^{2} u-\frac{Q_{\mathrm{n}}}{n}|u|^{2(n-1)} u+S, \\
\frac{\partial \phi_{\mathrm{c}}}{\partial \tau}=\theta_{\mathrm{c}_{\mathrm{n}}}|u|^{2 n}-\frac{\phi_{\mathrm{c}}}{\tau_{\mathrm{c}}}
\end{gathered}
$$

where $n=2$ in the case of 2PA is dominant, $n=3$ when 3PA is significant, and $n=4$ when $4 \mathrm{PA}$ is the dominant nonlinear loss mechanism. As an example, depending upon the bandgap energy of silicon (i.e., $E_{g} \sim 1.1 \mathrm{eV}$ ), only $2 \mathrm{PA}$, 3PA, or 4PA is significant at the operating wavelength $\lambda<2.2 \mu \mathrm{m}$ (telecommunication and near-IR wavelength), $\lambda>2.2 \mu \mathrm{m}$ to $\lambda<3.3 \mu \mathrm{m}$ (short-wavelength IR) and $\lambda>3.3 \mu \mathrm{m}$ (mid-IR), respectively, for silicon waveguides. Note that the operating wavelength range depends upon the doping concentration of the intrinsic material of the optical waveguide [44]. Free carriers are generated through multiphoton absorption that induces additional FCA losses to the system. FCA is associated with the FCD as free carriers are also able to modify the refractive index of the medium [41,44]. Particularly, if the intensity of the input incident pulse is very high, the effects of FCA and FCD will be crucial. However, in suitable conditions such as low pulse energy or in the presence of external bias that is able to sweep the free carriers, the effects of FCA-FCD can be neglected [41]. The carrier evolution given in (4) with respect to the slow time can be rewritten in the following form after including the boundary condition:

$$
\frac{\partial \phi_{\mathrm{c}}}{\partial \xi}=\theta_{\mathrm{c}_{\mathrm{n}}}\left(\frac{1}{t_{\mathbf{R}}} \int_{-\frac{\mathrm{R}_{\mathrm{R}}}{2}}^{\frac{t_{\mathrm{R}}}{2}}|u|^{2 n} d \tau\right)-\frac{\phi_{\mathrm{c}}}{\tau_{\mathrm{c}}} \approx \theta_{\mathrm{c}_{\mathrm{n}}}|u|^{2 n}-\frac{\phi_{\mathrm{c}}}{\tau_{\mathrm{c}}} .
$$

The approximation given in Eq. (7) works reasonably well at steady state or at the onset of both the self-pulsation (SP) and the MI. Realistic values of different parameters are taken from [41] and normalized [56]. In our simulations, the waveguide area and the effective area $\left(A_{\text {eff }}\right)$ are equivalents [41,57].

\section{STEADY-STATE SOLUTIONS}

We find the steady-state $(\partial u / \partial x=0)$, stationary $\partial u / \partial \tau=$ 0 ) homogeneous solutions of Eq. (5). In the steady state [58] the wave amplitude $u$ follows the relationship with the pump amplitude $S$ given by Eq. (8).

$$
u=\frac{S}{(1+i \Delta)+\frac{1}{2}(1+i K) \sum_{n=2}^{4} C_{\mathrm{n}}|u|^{2 n}-i|u|^{2}+\sum_{n=2}^{4} \frac{Q_{\mathrm{n}}}{n}|u|^{2(n-1)}} .
$$


If the intracavity power and input pump power are denoted by $Y\left(=|u|^{2}\right)$ and $X\left(=|S|^{2}\right)$, respectively, the steady-state, stationary, and homogeneous solution of LLE can be expressed by the characteristics polynomial of $Y$ having a degree of $(2 n+1)$ and can be written as Eq. (9) where $n$ varies from 2 to 4 if 2PA, 3PA, and 4PA all are present. Thus the characteristic polynomial satisfies a nonic polynomial of $Y$.

$$
\begin{aligned}
X= & \left(1+\Delta^{2}\right) Y-2 \Delta Y^{2}+Y^{3}+2 \sum_{n=2}^{4} \frac{Q_{\mathrm{n}}}{n} Y^{n}+(1+\Delta K) \sum_{n=2}^{4} C_{\mathrm{n}} Y^{n+1}-K \sum_{n=2}^{4} C_{\mathrm{n}} Y^{n+2}+\sum_{n=2}^{4} \frac{Q_{n}^{2}}{n^{2}} Y^{2 n-1} \\
& +\frac{1}{4}\left(1+K^{2}\right) \sum_{n=2}^{4} C_{\mathrm{n}}^{2} Y^{2 n+1}+\sum_{n=2}^{4} \sum_{q=2}^{4} \frac{C_{\mathrm{n}} Q_{\mathrm{q}}}{q} Y^{\mathrm{n}+\mathrm{q}} .
\end{aligned}
$$

In case only one out of 2PA, 3PA, and 4PA is dominant along with the FCA and FCD, Eq. (9) can be simplified as

$$
\begin{aligned}
X= & \left(1+\Delta^{2}\right) Y-2 \Delta Y^{2}+Y^{3}+\frac{2}{n} Q_{\mathrm{n}} Y^{n} \\
& +C_{\mathrm{n}}(1+\Delta K) Y^{n+1}-K C_{\mathrm{n}} Y^{n+2}+\frac{Q_{\mathrm{n}}^{2}}{n^{2}} Y^{2 n-1} \\
& +\frac{C_{\mathrm{n}} Q_{\mathrm{n}}}{n} Y^{2 n}+\frac{C_{\mathrm{n}}^{2}}{4}\left(1+K^{2}\right) Y^{2 n+1} .
\end{aligned}
$$

Equation (10) can further be reduced in Eq. (11) in the presence of only multiphoton absorption, whereas FCA and FCD are negligible.

$$
X=\left(1+\Delta^{2}\right) Y-2 \Delta Y^{2}+Y^{3}+\frac{2}{n} Q_{\mathrm{n}} Y^{n}+\frac{Q_{\mathrm{n}}^{2}}{n^{2}} Y^{2 n-1} .
$$

Equation (12) is the steady-state stationary solution of LLE while multiphoton absorption along with FCA is considered while the effect of FCD can be neglected.

$$
\begin{aligned}
X= & \left(1+\Delta^{2}\right) Y-2 \Delta Y^{2}+Y^{3}+\frac{2}{n} Q_{\mathrm{n}} Y^{n}+C_{\mathrm{n}} Y^{n+1} \\
& +\frac{Q_{\mathrm{n}}^{2}}{n^{2}} Y^{2 n-1}+\frac{C_{\mathrm{n}} Q_{\mathrm{n}}}{n} Y^{2 n}+\frac{C_{\mathrm{n}}^{2}}{4} Y^{2 n+1} .
\end{aligned}
$$

It is clearly visible from Eq. (12) that the steady-state, stationary solutions become quantic, septic, and a nonic polynomial of $Y$ in the presence of either 2PA, 3PA, or 4PA along with the corresponding FCA-FCD, respectively. Note that all these equations (9)-(12) are reduced to the well-known cubic polynomial of $Y$ if all the nonlinear losses are neglected [52], as given in Eq. (13). $C_{n}$ is defined as the product of $\theta_{c n}$ and the $\tau_{c}$. Thus, the polynomial of degree $(2 n+1>3)$ accounts for the steady-state homogeneous solutions of LLE for multiphoton absorptions in the presence of FCA and FCD.

$$
X=\left[\left(1+\Delta^{2}\right) Y-2 \Delta Y^{2}+Y^{3}\right] .
$$

\section{BISTABILITY AND KERR TILT}

Different important features such as the threshold pump power and threshold pump detuning that initiate the Turing pattern and eventually the stable comb formation can be retrieved from the bistability curve and the Kerr tilt which are obtained from the steady-state behavior of LLE [52-54]. In addition, the dynamics of FC can partially be understood through Kerr tilt and the bistability curve as the cavity soliton solutions of LLE result from the coexistence of patterned and CW solutions [58].

\section{A. Threshold condition to initiate optical bistability}

It is known that the minimum value of the normalized cavity detuning $\Delta$ that can initiate the optical bistability in the absence of all the nonlinear losses is $\sqrt{3}$ [52-54]. Multiphoton absorption along with FCA-FCD changes the threshold value of the cavity detuning. It is shown that in the presence of 2PA and FCA-FCD, the steady-state homogeneous solution of the LLE satisfies the quintic polynomial of $Y$. However, if the FCA and FCD are negligible, the polynomial reduces to a cubic polynomial having the $2 \mathrm{PA}$ coefficient $\left(Q_{2} \neq 0, C_{2}=0\right.$, $K=0$ ), which is given by

$$
X=\left(1+\Delta^{2}\right) Y+\left(Q_{2}-2 \Delta\right) Y^{2}+\left\{1+\frac{Q_{2}^{2}}{4}\right\} Y^{3} .
$$

In this case, as Eq. (14) is a cubic polynomial of $Y$, it is possible to find out an analytical expression of the threshold value of the normalized cavity detuning that initiates the optical bistability. Equating the $d X / d Y=0$, one can obtain the value of detuning beyond which the intracavity power will be multivalued, which is given by Eq. (15):

$$
\Delta_{ \pm} \geqslant \frac{8 Q_{2}}{4-3 Q_{2}^{2}} \pm \frac{\sqrt{3}}{4-3 Q_{2}^{2}}\left(Q_{2}^{2}+4\right)
$$

negative values of $\Delta$ being neglected. We calculate the threshold value of $\Delta$ from Eq. (15) and plot it in Fig. 1(a). It is observed that 2PA increases the threshold for optical bistability. We also find the saddle-node positions $X_{ \pm}$of the bistable curve by plugging the $\Delta_{+}$into Eq. (14).

$$
\begin{gathered}
X_{ \pm}= \pm \frac{4}{27}\left(\frac{1}{4+Q_{2}^{2}}\right)^{2}\left( \pm 4 \Delta \mp 2 Q_{2}+\sqrt{4-3 Q_{2}^{2}} \sqrt{\Theta}\right) \\
\times\left[\begin{array}{l}
12+Q_{2}^{2}+\Delta^{2}\left(4+3 Q_{2}^{2}\right) \pm Q_{2} \sqrt{4-3 Q_{2}^{2}} \sqrt{\Theta} \\
+2 \Delta\left(4 Q_{2} \mp \sqrt{4-3 Q_{2}^{2}} \sqrt{\Theta}\right)
\end{array}\right] \\
\Theta=\Delta^{2}-\frac{16 Q_{2}}{4-3 Q_{2}^{2}} \Delta+\frac{Q_{2}^{2}-12}{4-3 Q_{2}^{2}}
\end{gathered}
$$




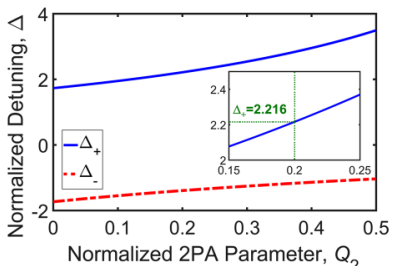

(a)

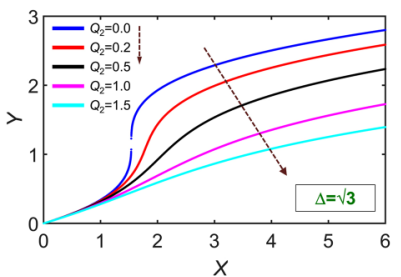

(c)

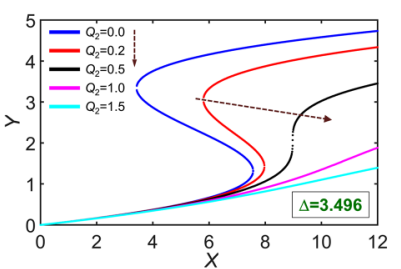

(e)

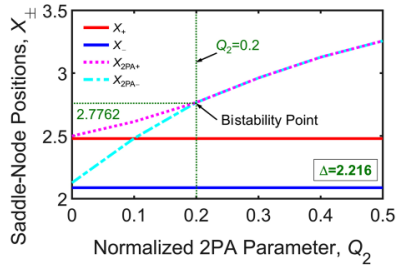

(b)

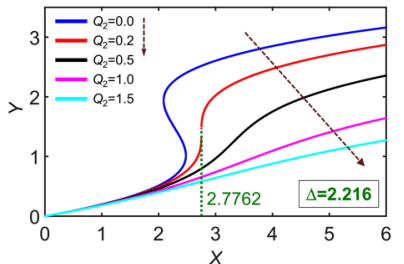

(d)

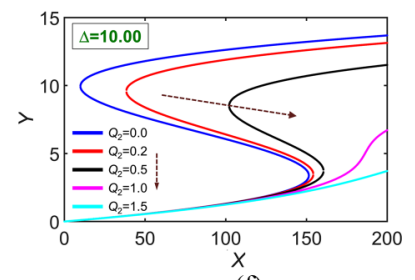

(f)
FIG. 1. Effect of 2PA on optical bistability. (a) Normalized detuning $\Delta$ vs normalized 2PA coefficient $Q_{2}$. (b) Saddle-node positions $X_{ \pm}$and $X_{2 \mathrm{PA} \pm}$ with $Q_{2}$ when $\Delta$ is fixed at 2.216 . Intracavity power $Y$ with the change in input pump power; $X$ for (c) $\Delta=\sqrt{3}$, (d) $\Delta=2.216$, (e) $\Delta=3.496$, and (f) $\Delta=10$, with a set of five different values of $Q_{2}$, in each case $\left(Q_{2}=0,0.2,0.5,1.0\right.$, and 1.5). Bistable behavior initiates at $\Delta=\sqrt{3}, 2.216$, and 3.496 when the $Q_{2}$ is $0,0.2$, and 0.5 , respectively. For $Q_{2}>2 \sqrt{3}$, bistability does not occur even if $\Delta$ is as high as $10.0(\mathrm{a}, \mathrm{f})$ with positive and realistic values of $X$ and $Y$.

In the absence of 2PA, Eq. (16) reduces to the known expression

$$
X_{ \pm}= \pm\left(\frac{2}{27}\right)\left( \pm 2 \Delta+\sqrt{\Delta^{2}-3}\right)\left(3+\Delta^{2} \mp \Delta \sqrt{\Delta^{2}-3}\right)
$$

The intracavity steady-state power $Y_{ \pm}$in the presence of 2PA can be given by

$$
\begin{gathered}
Y_{ \pm}=-\frac{1}{3} \frac{\left(Q_{2}-2 \Delta\right)}{\left(1+\frac{Q_{2}^{2}}{4}\right)} \pm \frac{1}{6} \frac{\sqrt{4-3 Q_{2}^{2}}}{\left(1+\frac{Q_{2}^{2}}{4}\right)} \sqrt{\Theta}, \\
Y_{ \pm}=\frac{1}{3}\left(2 \Delta \pm \sqrt{\Delta^{2}-3}\right)
\end{gathered}
$$

which reduces to the well-known Eq. (20) when $Q_{2}=0$. In Fig. 1(b), $X_{ \pm}$and $X_{2 \mathrm{PA} \pm}$ represent the value of normalized input pump power in the absence and presence of 2PA, respectively, at which the bistability starts (saddle nodes). In the absence of 2PA, both the curves [red (light gray) solid curve] for $X_{+}\left[X_{-}\right.$, blue (deep gray) solid curve] and the cyan (light gray) dashed-dotted curve for $X_{2 \mathrm{PA}+}\left[X_{2 \mathrm{PA}-}\right.$, dotted magenta (light gray)] initiate from the same point, as shown in Fig. 1(b). For $Q_{2}=0$, when the detuning $\Delta$ is 2.216 , both the

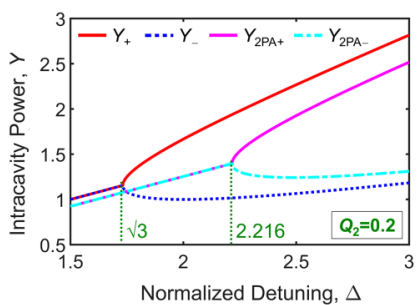

(a)

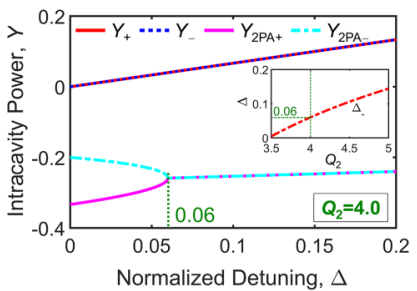

(c)

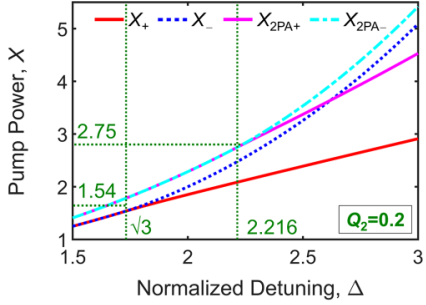

(b)

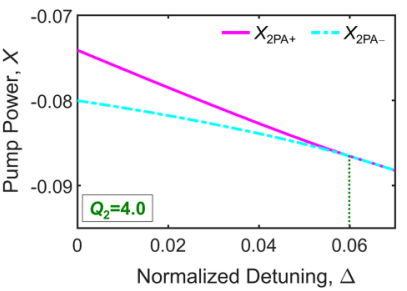

(d)
FIG. 2. The possible range of 2PA coefficients for which bistability can occur. Intracavity power $Y$ with respect to normalized detuning $\Delta$ at (a) $Q_{2}=0$, (c) $Q_{2}=4$. Input pump power $X$ vs $\Delta$, at (b) $Q_{2}=0$, (d) $Q_{2}=4.0$. Bistability occurs at a positive value of $Y$ and $X$ if $Q_{2}=0(<2 \sqrt{3})$, whereas bistability can be obtained for a very small value of $\Delta$, with negative values of $X$ and $Y$ when $\Delta=$ $4(>2 \sqrt{3})$.

values of $X_{+}$and $X_{-}$merge into 2.7762 , which indicates that if the $Q_{2}$ is large, bistability does not occur. As an example, we show that the 2PA increases the bistability threshold as presented in Figs. 1(c)-1(f). The arrow indicates the change in bistability pattern with the increase in $Q_{2}$.

If there is no 2PA, the bistability occurs at $\Delta=\sqrt{3}$. However, if the 2PA coefficient $Q_{2}$ is 0.2 and 0.5 , the minimum pump detunings that initiate the bistability are 2.216 and 3.496, respectively. Also, it can easily be found that, for any of the two roots $\left(\Delta_{+}\right.$or $\left.\Delta_{-}\right)$of Eq. (15) to be positive, the condition given by Eq. (21) has to be satisfied:

$$
Q_{2} \leqslant \frac{2}{\sqrt{3}} \text { or } Q_{2} \geqslant 2 \sqrt{3} \text {. }
$$

Earlier, it was predicted numerically that after a certain maximum value of 2PA coefficient bistability may cease to exist [59]. In contrast, our theoretical model predicts that it is possible to observe bistability at $Q_{2} \geqslant 2 \sqrt{3}$ with a very small detuning (even less than $\sqrt{3}$ ) [as shown in Figs. 2(a)-2(d)]. For Figs. 2(a) and 2(b), at first, we consider the case where $Q_{2}$ is less than $2 \sqrt{3}$ and show the values of intracavity and pump power for which the bistability initiates.

On the other hand, in Fig. 2(c) we plot the intracavity and pump power for which the bistability occurs when $Q_{2}>2 \sqrt{3}$. To obtain bistability in the latter case, the input pump power $X$ and intracavity power $Y$ both have to be negative, which is not possible in practice. Therefore, we restrict our analysis for the 2PA coefficient within $2 \sqrt{3}(\approx 1.154)$ and discard the other possibilities. It should also be noted that although optical bistability is a necessary condition for the formation of the optical cavity soliton, it is not a sufficient condition [59] to generate the cavity soliton. 


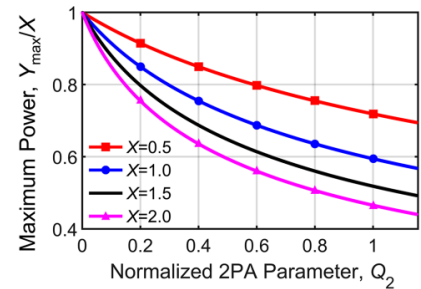

(a)

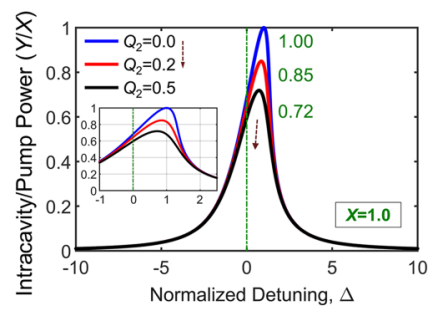

(c)

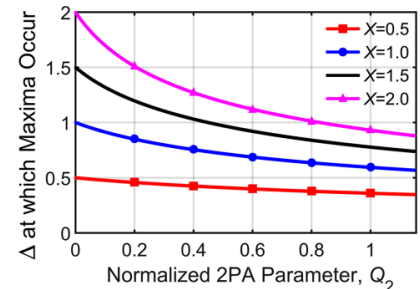

(b)

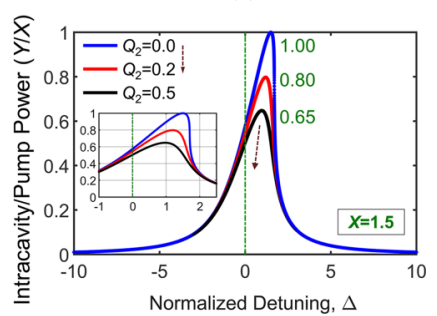

(d)
FIG. 3. Effect of 2PA on Kerr tilt. (a) The ratio of maximum normalized intracavity power $\left(Y_{\max }\right)$ and the normalized pump power $(X)$ with 2PA parameters, and (b) normalized detuning $(\Delta)$ with 2PA coefficient $\left(Q_{2}\right)$ where the maxima occur. Kerr tilts with different 2PA coefficients when normalized input power is (c) $X=1$ and (d) $X=1.5$.

\section{B. Kerr tilt with nonlinear losses}

Resonance of a cavity is tilted as a consequence of Kerr nonlinearity. In these circumstances, the resonance peak occurs at the normalized pump detuning $\Delta=Y=X$ [58]. Therefore, the real roots of Eq. (22), which can be obtained by putting $\Delta=Y$ in Eq. (11), yield the maximum value of $Y$, i.e., $Y_{\max }$ for a particular normalized pump power, $X$ in the presence of 2PA $(n=2)$.

$$
\frac{Q_{\mathrm{n}}^{2}}{n^{2}} Y^{2 n-1}+\frac{2}{n} Q_{\mathrm{n}} Y^{n}+Y-X=0 .
$$

The ratio of normalized intracavity power with respect to the normalized pump power (i.e., $Y_{\max } / X$ ) and the corresponding normalized detuning $\Delta$ at which the maxima occur are plotted in Figs. 3(a) and 3(b), respectively, for different values of normalized 2PA coefficients $Q_{2}(0-0.5)$.

Figures 3(c) and 3(d) show the Kerr tilt for three distinct values of $Q_{2}(0,0.2$, and 0.5$)$ at two different input pump powers $(X=1$ and 1.5$)$. It is apparent that the higher the input pump power, greater is the slope of the Kerr tilt, which means one requires larger external pump detuning to obtain maximum intracavity power. In addition, if the 2PA is present, with the increase in input pump power, intracavity power decreases as the nonlinear absorption is more prominent at comparatively high input power.

\section{Bistability and Kerr tilt in the presence of FCA-FCD}

In the previous section, we have provided a detailed quantitative analysis of the shift in Kerr tilt as well as the reduction in the intracavity output power in the presence of 2PA.

In this section, we discuss the optical bistability and Kerr tilt in the presence of FCA-FCD. To investigate the effect of FCA-FCD, we have assumed arbitrary values of FCA and FCD coefficients. The bistability curves at a fixed pump

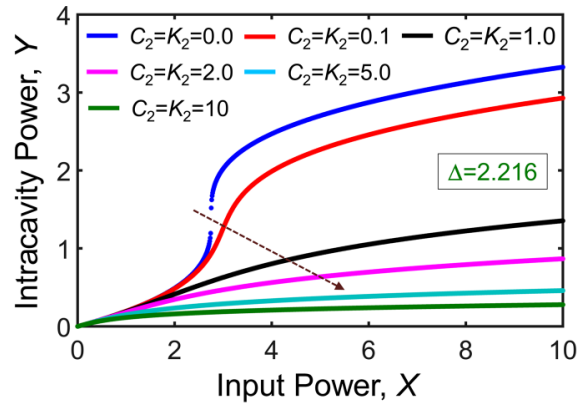

(a)

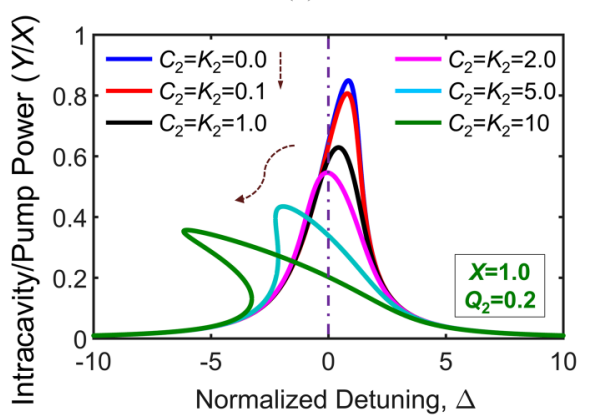

(b)

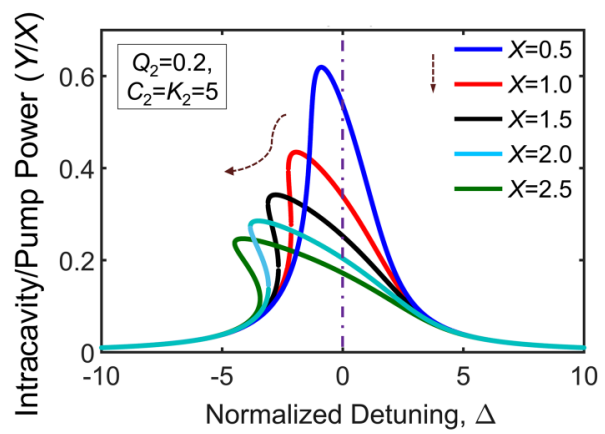

(c)

FIG. 4. (a) Bistability curve at $\Delta=2.216$, and (b) Kerr tilt at $X=1$, for a set of six different values of $2 \mathrm{PA}$-induced FCA-FCD ( $C_{2}$ and $K_{2}$, respectively). (c) Dependence of Kerr tilt on input pump power $X$ when $C_{2}=K_{2}=5$. For all the cases, the $2 \mathrm{PA}$ coefficient $Q_{2}$ is taken as 0.2 .

detuning are plotted in Fig. 4(a) for different values of FCA and FCD coefficients. Figures 4(b) and 4(c) exhibit the Kerr tilt as a function of pump detuning. FCA-induced loss has a similar effect on the bistability curve as that of the multiphoton absorption. It is also evident that FCD introduces a cavity detuning that manifests itself through the reverse Kerr tilt. In practice, the reverse Kerr tilt shown in Fig. 4(b) indicates that with suitable FCD-induced cavity detuning, one can obtain maximum intracavity power even without any external pump detuning (solid pink curve). For a fixed value of FCA-FCD coefficient, the reverse tilt increases with the increase in the input pump power $X$, as depicted in Fig. 4(c). It is seen that the intracavity power becomes multivalued with large detuning and pump power. A similar kind of reverse Kerr tilt as shown in Figs. 4(b)-4(d) is also reported in a recently published manuscript that discusses the possibility of parametric oscillations in silicon microcavities in the presence of 2PA [60]. 


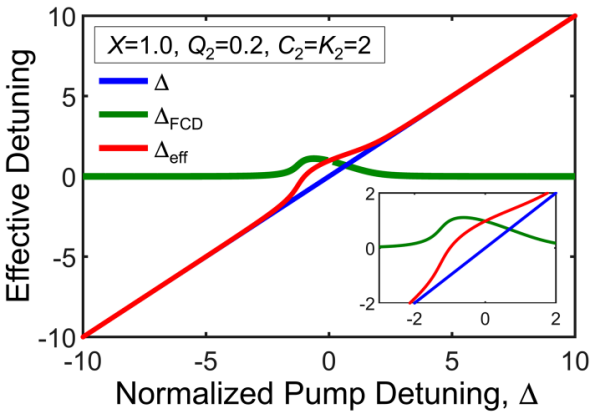

(a)

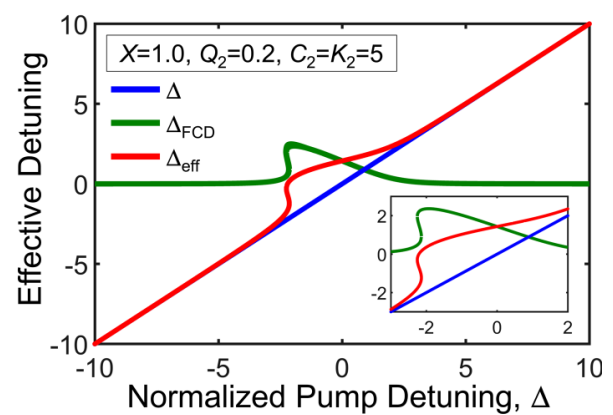

(b)

FIG. 5. Effective detuning $\Delta_{\text {eff }}$ [diagonal, red (light gray) curve] and FCD-induced detuning $\Delta_{\mathrm{FCD}}$ [horizontal, green (light gray) curve], with the variation in pump detuning when (a) $C_{2}=K_{2}=2$, and (b) $C_{2}=K_{2}=5$ at input pump power; $X=1$ in both cases. Input pump detuning is also plotted [blue (deep gray) curve] in $(a, b)$ to indicate the reference level in the absence of FCA-FCD. $\Delta_{\mathrm{FCD}}$ can possess multiple values if FCA-FCD coefficients are high.

\section{FCA-FCD-induced cavity detuning}

To explain the reverse Kerr tilt analytically, we further modify the normalized LLE given in Eq. (5) as

$$
\begin{gathered}
\frac{\partial u}{\partial \xi}=-\left(1+i \Delta_{\text {eff }}\right) u-\frac{1}{2} \phi_{\mathrm{c}} u-i \frac{s}{2} \frac{\partial^{2} u}{\partial \tau^{2}} \\
+\sum_{k \geqslant 3} \frac{d_{\mathrm{k}}}{k !} \frac{\partial^{k} u}{\partial \tau^{k}}+i|u|^{2} u-\frac{\mathrm{Q}_{\mathrm{n}}}{n}|u|^{2(n-1)} u+S, \\
\Delta_{\text {eff }}=\Delta+\Delta_{\mathbf{F C D}}=\Delta+\frac{K \phi_{c}}{2}
\end{gathered}
$$

where the effective cavity detuning $\Delta_{\text {eff }}$ is defined as the sum of the external pump detuning $\Delta$ and the FCD-induced detuning $\left(D_{\mathrm{FCD}}\right)$. It should be noted that the $\Delta_{\text {eff }}$ changes over round trips. Effective detuning $\Delta_{\text {eff }}$ [diagonal, solid red (light gray) curve] and FCD-induced detuning $\Delta_{\mathrm{FCD}}$ [horizontal, solid green (light gray) curve], for two sets of FCA-FCD coefficients, $C_{2}=K_{2}=2$, and $C_{2}=K_{2}=5$, at input pump power $X=1$ are plotted in Figs. 5(a) and 5(b), respectively. In both cases, input pump detuning has also been plotted [diagonal, solid blue (deep gray) curve] to indicate the reference level in the absence of FCA-FCD. Figures 5(a) and 5 (b) show how the overall cavity detuning is affected by the FCD-induced detuning at different FCA-FCD coefficients. The intersection between the diagonal, red (light gray) and

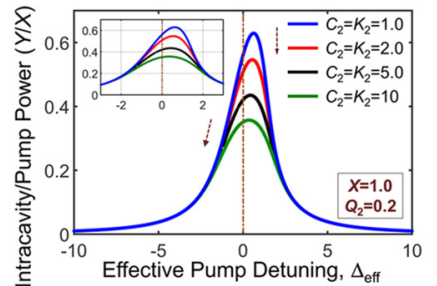

(a)

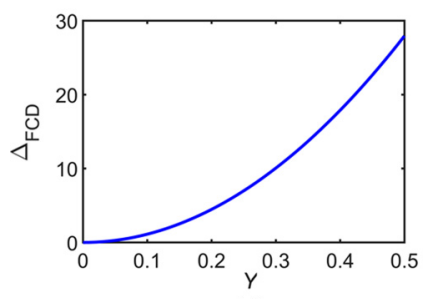

(c)

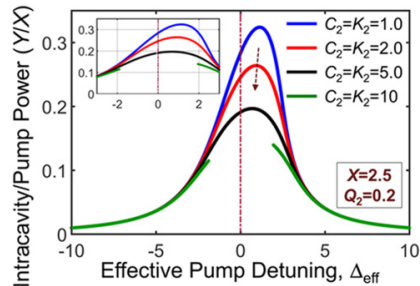

(b)

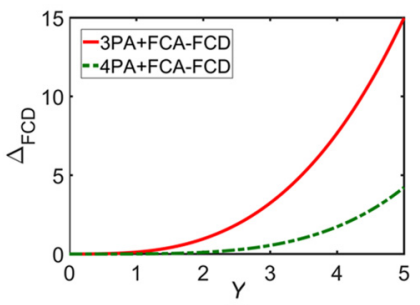

(d)
FIG. 6. Kerr tilt $(Y / X)$ with the change in effective detuning, $\Delta_{\text {eff }}$, instead of pump detuning, $\Delta$ for two different pump powers: (a) $X=$ 1 and (b) $X=2.5$. FCD-induced steady-state cavity detuning, $\Delta_{\mathrm{FCD}}$ with the intracavity power, $Y$ when the free carriers are generated due to the (c) 2PA [blue (deep gray) solid curve], (d) 3PA [red (light gray) solid curve], and 4PA [green (light gray) dotted curve].

the horizontal, green (light gray) curves indicates the effective cavity detuning when the external pump detuning is zero. Generation of a stable frequency comb even without external pump detuning with suitable initial pump power has been previously demonstrated [47].

Note that, in order to excite the temporal cavity soliton in the microresonator experimentally, one must modulate the phase of the microresonator driving field which can be done through electrical or thermal detuning. Recently, the effect of thermo-optical chaos on soliton generation was studied both numerically and experimentally [61]. Thermal detuning can easily be included in our numerical model following the approach adopted in [61]. In this work, however, we assume that the microresonator is in a thermal equilibrium state and neglect such thermally induced detuning while studying the steady-state behavior and the MI in the presence of all nonlinear losses.

We also plot the normalized intracavity power $(Y / X)$ with the change in $\Delta_{\text {eff }}$ instead of $\Delta$ in Figs. 6(a) and 6(b) for two different values of $X$. As expected, this time there is no tilt in the opposite direction. When the carrier accumulation reaches the steady state, the effective detuning, $\Delta_{\text {eff }}$ can be written as

$$
\Delta_{\text {eff }}^{(\text {Steady state })}=\Delta+\frac{K C_{\mathrm{n}}\left|u_{0}\right|^{2 n}}{2}=\Delta+\frac{K C_{\mathrm{n}} Y_{0}{ }^{n}}{2},
$$

where $u_{0}$ is the steady-state amplitude and the $Y_{0}$ is the steadystate intracavity power. The steady-state FCD-induced cavity detuning for 2PA, as plotted in Fig. 6(c), is much larger than the FCD-induced cavity detuning due to the 3PA and 4PA as shown in Fig. 6(d).

We have considered realistic values of FCA-FCD coefficients calculated from $[41,56]$ in order to obtain the steadystate maximum FCD-induced cavity detuning for 2PA, 3PA, and 4PA as shown in Figs. 6(c) and 6(d). 


\section{GENERALIZED EXPRESSION: BISTABILITY AND SELF-PULSATION}

The stability of Eq. (10) can be analyzed by perturbing the steady-state solutions $u_{0}$ and $\phi_{0}$ with small variations in the amplitude $\delta u(\xi)$ and the free carriers $\delta \phi_{c}(\xi)$, with respect to the slow time, respectively. Thus if $u(\xi)=u_{0}+$ $\delta u(\xi)$ and $\phi_{c}(\xi)=\phi_{0}+\delta \phi_{c}(\xi)$, then the perturbation array $\varepsilon=\left(\delta u, \delta u^{*}, \delta \phi_{c}\right)^{T}$ in the presence of nPA and corresponding FCA-FCD is found to satisfy the following linearized equation:

$$
\frac{d \varepsilon}{d \xi}=J_{\mathbf{B S}, \mathbf{S P}}^{(\mathrm{n})} \varepsilon,
$$

where $J_{\mathbf{B S}, \mathbf{S P}}^{(\mathrm{n})}$ is the $3 \times 3$ Jacobian matrix of (10) and (11) and is given by

$$
J_{\mathbf{B S}, \mathbf{S P}}^{(\mathrm{n})}=\left(\begin{array}{ccc}
R_{\mathbf{B S}, \mathbf{S P}}^{(\mathrm{n})}+i I_{\mathbf{B S}, \mathbf{S P}}^{(\mathrm{n})} & -\left(\frac{n-1}{n} Y^{n-2} Q_{\mathrm{n}}-i\right) u_{0}^{2} & -\frac{1}{2}(1+i K) u_{0} \\
-\left(\frac{n-1}{n} Y^{n-2} Q_{\mathbf{n}}+i\right) u_{0}^{* 2} & R_{\mathbf{B S}, \mathbf{S P}}^{(\mathrm{n})}-i I_{\mathbf{B S}, \mathbf{S P}}^{(\mathrm{n})} & -\frac{1}{2}(1-i K) u_{0}^{*} \\
n Y^{n-1} u_{0}^{*} \theta_{\mathrm{c}_{\mathrm{n}}} & n Y^{n-1} u_{0} \theta_{\mathrm{c}_{\mathrm{n}}} & -\frac{1}{\tau_{\mathrm{c}}}
\end{array}\right),
$$

where $R_{\mathbf{B S}, \mathbf{S P}}^{(\mathrm{n})}$ and $I_{\mathbf{B S}, \mathbf{S P}}^{(\mathrm{n})}$ are

$$
\begin{gathered}
R_{\mathbf{B S}, \mathbf{S P}}^{(\mathrm{n})}=-\left[1+\frac{C_{\mathrm{n}}}{2} Y^{n}+Q_{n} Y^{(n-1)}\right], \\
I_{\mathbf{B S}, \mathbf{S P}}^{(\mathrm{n})}=-\left[\Delta+\frac{K C_{\mathrm{n}}}{2} Y^{n}-2 Y\right] .
\end{gathered}
$$

The conditions for BS and SP (free-carrier oscillation) can be obtained from $J_{\mathbf{B S}, \mathbf{S P}}^{(\mathrm{n})}$ in the presence of FCA-FCD which can be written in a general form as

$$
\begin{gathered}
\mathrm{BS}: \operatorname{det}\left(J_{\mathbf{B S}, \mathbf{S P}}^{(\mathrm{n})}\right)>0, \\
\mathrm{SP}:\left[\left\{\operatorname{tr}\left(J_{\mathbf{B S}, \mathbf{S P}}^{(\mathrm{n})}\right)^{2}-\operatorname{tr}\left(J_{\mathbf{B S}, \mathbf{S P}}^{(\mathrm{n}) 2}\right)\right\} \operatorname{tr}\left(J_{\mathbf{B S}, \mathbf{S P}}^{(\mathrm{n})}\right)\right. \\
\left.-2 \operatorname{det}\left(J_{\mathbf{B S}, \mathbf{S P}}^{(\mathrm{n})}\right)\right]>0,
\end{gathered}
$$

where det and $\operatorname{tr}$ are the determinant and the trace, respectively. Equation (31) corresponds to the Hopf bifurcation.

\section{LINEAR STABILITY ANALYSIS}

In this section, we perform the linear stability analysis of the stationary $\mathrm{CW}$ solutions $\left(u_{0}\right.$ and $\left.\phi_{0}\right)$ of free-carrier-driven LLE. The evolution of normalized carrier density and signal amplitude due to the spatiotemporal perturbations are given by Eqs. (32) and (33), respectively, in the presence of nonlinear losses including FCA-FCD. In each case, we find the MI gain $\lambda$ with the normalized sideband frequency $\Omega$. We also show the dependence of MI gain and bandwidth on normalized detuning $\Delta$, signal power $Y$, and higher-order dispersion. Finally, we provide a general expression of MI gain for multiphoton absorptions. Throughout our manuscript superscript (n) stands for nPA and unlike the powers, the super- and subscripts are not italic.

$$
\begin{gathered}
\phi_{\mathbf{c}}(\xi) \approx \phi_{0}(\xi)+\delta \phi(\xi), \\
u(\xi, \tau)=u_{0}(\xi)+u_{+}(\xi) e^{i \Omega \tau}+u_{-}(\xi) e^{-i \Omega \tau} .
\end{gathered}
$$

The carriers cannot follow the quick oscillation of the optical field and therefore, unlike the optical field amplitude $u$, the carrier density cannot change in fast-time $\tau$. In the presence of nPA at the onset of the MI, the carrier density is equal to $\phi_{0}$. Substituting the $\phi_{c}$ and $u$ given by Eqs. (32) and (33) into Eqs. (3) and (4), respectively, the general expressions for homogeneous solutions for the normalized free carrier and the intracavity field amplitude $\left(\phi_{0}, u_{0}\right.$, respectively) can be obtained as

$$
\begin{gathered}
\phi_{0}=\theta_{\mathbf{c}_{\mathbf{n}}} \tau_{\mathbf{c}}\left|u_{0}\right|^{2 n}=C_{\mathbf{n}}\left|u_{0}\right|^{2 n}, \\
\frac{\partial u_{0}}{\partial \xi}=-(1+i \Delta) u_{0}-\frac{1}{2}(1+i K) \theta_{\mathbf{c}_{\mathbf{n}}} \tau_{\mathbf{c}}\left|u_{0}\right|^{2 n} u_{0} \\
+i\left|u_{0}\right|^{2} u_{0}-\frac{Q_{\mathbf{n}}}{n}\left|u_{0}\right|^{2(n-1)} u_{0}+S,
\end{gathered}
$$

whereas the perturbations signal amplitudes $\left(u_{+}\right.$and $\left.u_{-}^{*}\right)$ are written as

$$
\begin{aligned}
\frac{\partial u_{+}}{\partial \xi}= & -(1+i \Delta) u_{+}-\frac{1}{2}(1+i K) \phi_{0} u_{+}+i \frac{s}{2} \Omega^{2} u_{+} \\
& -i \frac{d_{3}}{6} \Omega^{3} u_{+}+i\left(2\left|u_{0}\right|^{2} u_{+}+\left(u_{0}\right)^{2} u_{-}^{*}\right) \\
& -\frac{Q_{\mathbf{n}}}{n}\left(n\left|u_{0}\right|^{2(n-1)} u_{+}+(n-1)\left|u_{0}\right|^{2(n-2)}\left(u_{0}\right)^{2} u_{-}^{*}\right), \\
\frac{\partial u_{-}^{*}}{\partial \xi}= & -(1-i \Delta) u_{-}^{*}-\frac{1}{2}(1-i K) \phi_{0} u_{-}^{*}-i \frac{s}{2} \Omega^{2} u_{-}^{*} \\
& +i \frac{d_{3}}{6} \Omega^{3} u_{-}^{*}-i\left(2\left|u_{0}\right|^{2} u_{-}^{*}+\left(u_{0}^{*}\right)^{2} u_{+}\right) \\
& -\frac{Q_{\mathbf{n}}}{n}\left(n\left|u_{0}\right|^{2(n-1)} u_{-}^{*}+(n-1)\left|u_{0}\right|^{2(n-2)}\left(u_{0}^{*}\right)^{2} u_{+}\right) .
\end{aligned}
$$

Note that for the weak amplitudes and exponentially growing solutions, $u_{-}^{*}=u_{+}$. The Jacobian, $J_{\mathrm{MI}}$, to find the MI growth rate (gain) can be written as

$$
\begin{aligned}
J^{\mathbf{M I}} & =\left[\begin{array}{cc}
\frac{\partial}{\partial u_{+}}\left(\frac{\partial u_{+}}{\partial \xi}\right) & \frac{\partial}{\partial u_{-}^{*}}\left(\frac{\partial u_{+}}{\partial \xi}\right) \\
\frac{\partial}{\partial u_{+}}\left(\frac{\partial u_{-}^{*}}{\partial \xi}\right) & \frac{\partial}{\partial u_{-}^{*}}\left(\frac{\partial u_{-}^{*}}{\partial \xi}\right)
\end{array}\right] \\
& =\left[\begin{array}{cc}
J_{11}^{\mathbf{M I}} & J_{12}^{\mathbf{M I}}\left(u_{0}\right)^{2} \\
J_{21}^{\mathbf{M I}}\left(u_{0}^{*}\right)^{2} & J_{22}^{\mathbf{M I}}
\end{array}\right] .
\end{aligned}
$$


The eigenvalues of the matrix $J^{\mathrm{MI}}$ yield the modulation instability growth rate $\lambda$, which is calculated by equating the $\operatorname{det}\left(J^{\mathrm{MI}}-\lambda . I\right)=0$ where $I$ is a $2 \times 2$ identity matrix. The growth of the sideband amplitude can be expressed as $u_{+}=$ $\operatorname{Cexp}(\lambda \xi)$ where $C$ is an arbitrary constant. One can write $J_{p q}^{\mathrm{MI}}$ in the following form:

$$
J_{p q}^{\mathrm{MI}}=\left\{\begin{array}{c}
R_{\mathbf{M I}}^{(\mathbf{n})}+(-1)^{(q-1)} i I_{\mathbf{M I}}^{(\mathbf{n})}, \text { when } p=q \\
\left(-\alpha_{\mathbf{M I}}^{(\mathbf{n})}\right)+(-1)^{(q)} i\left(-\beta_{\mathbf{M I}}^{(\mathbf{n})}\right), \text { when } p \neq q
\end{array} .\right.
$$

The MI gain can be obtained by solving the quadratic equation (39) of $\lambda$ only for the real values:

$$
\left(\frac{\left\{R_{\mathbf{M I}}^{(\mathbf{n})}-\lambda\right\}^{2}+\left\{I_{\mathbf{M I}}^{(\mathbf{n})}\right\}^{2}}{\left\{\alpha_{\mathbf{M I}}^{(\mathbf{n})}\right\}^{2}+\left\{\beta_{\mathbf{M I}}^{(\mathbf{n})}\right\}^{2}}-Y^{2}\right)=0 .
$$

Here, the LLE is truncated up to the third-order dispersion. $R_{\mathrm{MI}}{ }^{(\mathrm{n})}, I_{\mathrm{MI}}{ }^{(\mathrm{n})}, a^{(\mathrm{n})}$, and $\beta^{(\mathrm{n})}$ are given as

$$
\begin{gathered}
R_{\mathbf{M I}}^{(\mathbf{n})}=-\left[1+\frac{C_{\mathbf{n}}}{2} Y^{n}+Q_{n} Y^{(n-1)}\right], \\
I_{\mathbf{M I}}^{(\mathbf{n})}=-\left[\Delta+\frac{K C_{\mathbf{n}}}{2} Y^{n}-\frac{s}{2} \Omega^{2}+\frac{d_{3}}{6} \Omega^{3}-2 Y\right], \\
\alpha_{\mathbf{M I}}^{(\mathbf{n})}=\left[\frac{n-1}{n} Q_{\mathbf{n}} Y^{(n-2)}\right], \\
\beta_{\mathbf{M I}}^{(\mathbf{n})}=-1 .
\end{gathered}
$$

Equation (39) can be simplified in a more convenient form given by Eq. (44):

$$
\left[\lambda^{2}-2 R_{\mathbf{M I}}^{(\mathbf{n})} \lambda+R_{\mathbf{M I}}^{(\mathbf{n})^{2}}+I_{\mathbf{M I}}^{(\mathbf{n})^{2}}-\left(\alpha_{\mathbf{M I}}^{(\mathbf{n})^{2}}+\beta_{\mathbf{M I}}^{(\mathbf{n})^{2}}\right) Y^{2}\right]=0 .
$$

Note that, in the absence of all nonlinear losses (multiphoton absorption, FCA, FCD) and the higher-order dispersion terms, $R_{\mathrm{MI}}{ }^{(n)}, I_{\mathrm{MI}}{ }^{(n)}, a_{\mathrm{MI}}{ }^{(n)}$, and $\beta_{\mathrm{MI}}{ }^{(n)}$ become $-1,-(\Delta-$ $\left.s \Omega^{2} / 2-2 Y\right), 0$, and -1 , respectively, such that $\lambda$ reduces to the well-known expression (45) for MI gain [53,54]:

$$
\lambda_{ \pm}(\Omega)=-1 \pm \sqrt{4 Y\left(\Delta-\frac{s \Omega^{2}}{2}\right)-\left(\Delta-\frac{s \Omega^{2}}{2}\right)^{2}-3 Y^{2}} .
$$

\section{A. MI in normal dispersion regime $(s=+1)$}

It is known that unlike a straight waveguide or optical fiber, MI can occur in synchronously driven optical cavities or in ring lasers even if the system is pumped in the normal dispersion regime [53]. As a result, a stable stationary train of pulses can be generated in the cavity irrespective of the sign of the dispersion.

In this section, we discuss the dependency of MI gain $\lambda$ on $\Omega$ and $\Delta$ (at $Y=2.5$ ) as well as on $\Omega$ and $Y$ (at $\Delta=7.5$ ), respectively, in the normal dispersion region. At first, we plot MI gain, $\lambda_{ \pm}$, with respect to normalized frequency $\Omega(>0)$ in Fig. 7(a). It is seen that 2PA in silicon can completely inhibit the parametric oscillations. Figure 7(b) shows the MI gain in the presence of 3PA. Nonzero third-order dispersion

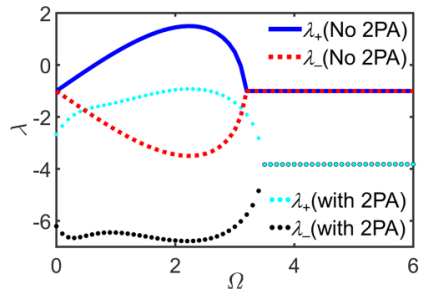

(a)

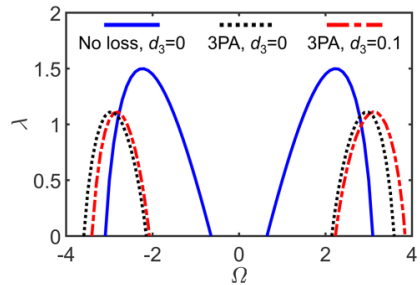

(b)
FIG. 7. (a) MI-gain lobes $\lambda_{ \pm}$in the absence and presence of $2 \mathrm{PA}$ for $\Omega>0$, (b) $\lambda_{+}$in the presence and absence of 3PA. Simulation parameters are $s=1, Y=2.5, D=7.5, Q_{2}=1, K_{2}=0.05, C_{2}=$ $0.1, \theta_{c 2}=0.05, \theta_{c 3}=6.31 \times 10^{-7}, Q_{3}=8.73 \times 10^{-4}, C_{3}=0.049$, $K_{3}=4.9$. Effect of third-order dispersion $\left(d_{3}=0.1\right)$ is also considered in (b) [red (light gray) dashed curve]. Third-order dispersion induces asymmetry in the gain lobe with respect to $\Omega=0$.

$\left(d_{3}=0.1\right)$ induces the asymmetry in the MI-gain lobe with respect to $\Omega=0$ and thereby enhances the MI bandwidth [46]. The values of nPA and FCA-FCD coefficients are taken from [41].

Figures 8(a) and 8(b) depict the dependency of MI gain $\lambda$ on $\Omega$ and $\Delta($ at $Y=2.5)$ as well as on $\Omega$ and $Y($ at $\Delta=7.5$ ), respectively, in the normal dispersion region while all the nonlinear losses are ignored.

To produce the rest of the curves [Figs. 8(c)-8(h)] we use the realistic values of $\mathrm{nPA}$ and FCA-FCD coefficients for silicon $(c-\mathrm{Si})$ waveguides from $[41,56]$. In the presence of 2PA no parametric gain lobe has been observed [Figs. 8(c) and 8(d)]. The MI gain in the presence of 3PA, 4PA, and the corresponding FCA-FCD is plotted in Figs. 8(e)-8(h). The cyan and magenta lines are drawn on the two-dimensional (2D) plots to indicate the corresponding values of $\lambda$ for $\Delta=$ 7.5 and $Y=2.5$, respectively [53]. Projection of the $2 \mathrm{D}$ plot on each axis through the shadow plot helps to anticipate the approximate values of the MI gain with respect to different parameters.

\section{B. MI in anomalous dispersion regime $(s=-1)$ and the effect of FCA-FCD on MI-growth rate}

Similar curves can be plotted for the MI gain in case of the anomalous dispersion regime of operation. We observe there is no parametric gain in the presence of 2PA at telecom wavelengths. It was previously reported by Lau et al. [41] that in mid-infrared (MIR) while the dominant loss mechanism is 3PA, the effect of 3PA-induced FCA-FCD is prominent and the parametric oscillations can only take place if the pump power is sufficiently low [41]. In their simulations, the waveguide is pumped at the anomalous region $(s=-1)$. This claim can be supported analytically through the MI analysis in the presence of nonlinear losses as shown in Fig. 9. We plot the $\lambda$ with $\Omega$ and $\Delta$ in Fig. 9(a) in the absence of any nonlinear losses, whereas Fig. 9(b) dictates the MI gain in the absence of FCA-FCD while 3PA is present. Figure 9(c) shows the MI gain in the presence of 3PA and FCA-FCD at $Y=2.5$. Similarly, when the intracavity power is almost quadrupled $(Y=10.5)$ the corresponding MI gain has been plotted in Figs. 9(d)-9(f) for three different cases as described earlier. It is conspicuous from Figs. 9(c) and 9(f) that there 


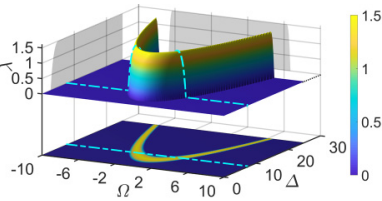

(a)

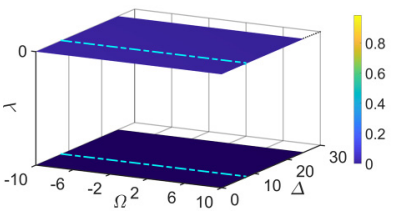

(c)

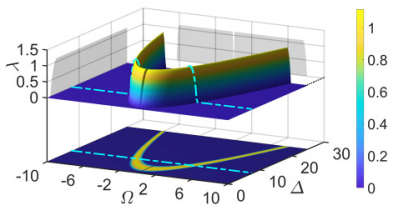

(e)

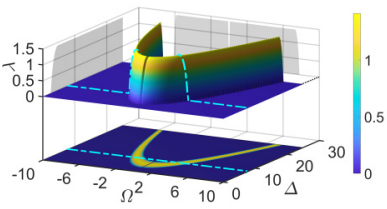

(g)

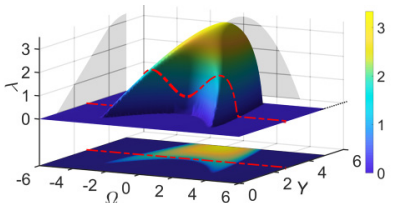

(b)

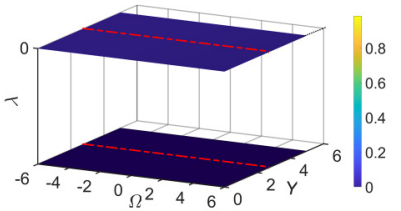

(d)

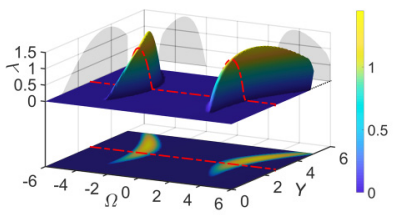

(f)

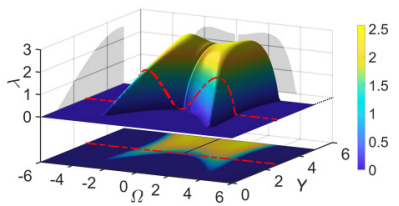

(h)
FIG. 8. Real part of the MI gain $\lambda$ vs (a) $\Omega$ and $\Delta$ (at $Y=$ 2.5), and (b) $\Omega$ and $Y$ (at $\Delta=7.5$ ) in the absence of all nonlinear losses. Variation of $\lambda$ with (c) $\Omega$ and $\Delta$ (at $Y=2.5$ ), (d) $\Omega$ and $Y$ (at $\Delta=7.5$ ) while $2 \mathrm{PA}$ and FCA-FCD are present. Change in $\lambda$ with the change in (e) $\Omega$ and $\Delta$ (at $Y=2.5$ ), and (f) $\Omega$ and $Y$ (at $\Delta=7.5$ ) when 3PA and FCA-FCD are present. $\lambda$ with $(\mathrm{g}) \Omega$ and $\Delta($ at $Y=2.5)$, and (h) $\Omega$ and $Y$ (at $\Delta=7.5$ ) in the presence of 4PA and FCA-FCD. For all the cases, third-order dispersion $\left(d_{3}=0.1\right)$ is taken into consideration. Other simulation parameters are $s=1, \theta_{c 2}=0.0005, Q_{2}=0.93, C_{2}=29.81, K_{2}=7.5\left(\right.$ at $\lambda_{0} \sim$ $1.56 \mu \mathrm{m}) ; \theta_{c 3}=6.31 \times 10^{-7}, Q_{3}=8.73 \times 10^{-4}, C_{3}=0.049, K_{3}=$ $4.9\left(\right.$ at $\left.\lambda_{0} \sim 2.4 \mu \mathrm{m}\right) ; \theta_{c 4}=6.018 \times 10^{-8}, Q_{4}=6.16 \times 10^{-6}, C_{4}=$ $4.66 \times 10^{-3}, K_{4}=2.9$ (at $\lambda_{0} \sim 4.0 \mu \mathrm{m}$ ). The cyan and magenta lines overlaid on the $2 \mathrm{D}$ plots indicate the corresponding values of $\lambda$ for $\Delta=7.5$ and $Y=2.5$, respectively.

is no parametric oscillation if FCA-FCD is present while the intracavity power is high $(Y=10.5)$; however, MI gain lobes exist for relatively low intracavity power $(Y=2.5)$. Therefore, in the case of 3PA, the principle mechanisms to inhibit the comb formation are 3PA-induced FCA and FCD [41]. To obtain parametric oscillation in the presence of 3PA, either the generated free carriers have to be swept away by suitable external bias or the input pump power $X$ (consequently, $Y$ ) has to be sufficiently low.

\section{CONDITION FOR MAXIMUM MI GAIN AND THRESHOLD INTENSITY}

It is known [54] that the MI gain in the absence of nonlinear losses can be given by Eq. (46) while the maximum gain

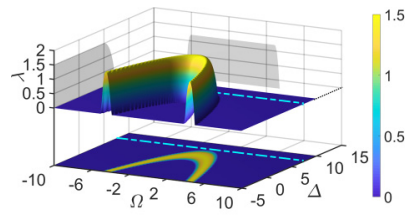

(a)

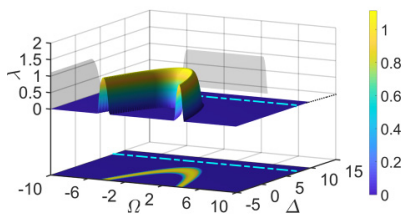

(c)

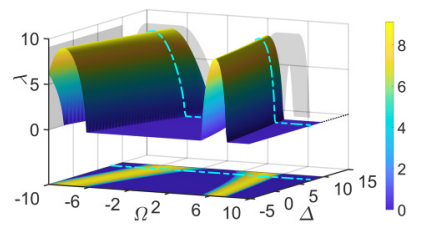

(e)

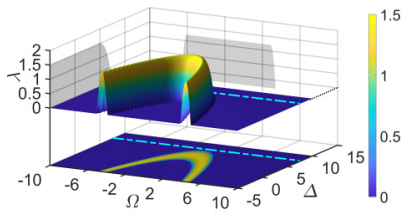

(b)

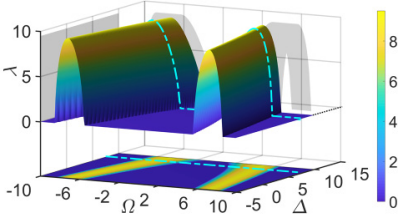

(d)

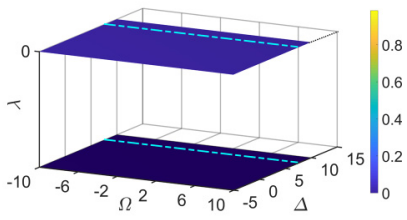

(f)
FIG. 9. MI gain $\lambda$ with respect to $\Omega$ and $\Delta$ when $Y$ is fixed at 2.5 in (a) the absence of all nonlinear losses, (b) the presence of only 3PA and no FCA-FCD, and (c) the presence of 3PA and FCA-FCD. MI gain $\lambda$ with respect to $\Omega$ and $\Delta$ when at $Y=10.5$ in (d) the absence of all nonlinear losses, (e) the presence of only 3PA and no FCA-FCD, and (f) the presence of 3PA, FCA-FCD. It is observed that if input power $X$ is high (high intracavity power, $Y$ ) parametric oscillation ceases to occur in the presence of FCA-FCD along with 3PA.

$\left(\lambda=\lambda_{\max }=Y-1\right)$ can be achieved if $\Delta k=0$,

$$
\lambda_{ \pm}(\Omega)=-1 \pm \sqrt{Y^{2}-(\Delta k)^{2}}
$$

where $\Delta k$ is given by

$$
\Delta k=\left(\Delta-\frac{s}{2} \Omega^{2}-2 Y\right) .
$$

Therefore, the steady-state solution will always be stable if $Y<1$. However, in the presence of nonlinear losses, the solution of $\lambda$ can be written as (48) instead of Eq. (47).

$$
\lambda^{(\mathbf{n})}=R_{\mathbf{M I}}^{(\mathbf{n})} \pm \sqrt{\left\{\left(\alpha_{\mathbf{M I}}^{(\mathbf{n})}\right)^{2}+\left(\beta_{\mathbf{M I}}^{(\mathbf{n})}\right)^{2}\right\} Y^{2}-\left(I_{\mathbf{M I}}^{(\mathbf{n})}\right)^{2}} .
$$

In this case, the maximum gain can be obtained if $I_{\mathbf{M I}}^{(\mathbf{n})}=0$. One can write the general expression of $\lambda_{\max }$ in terms of $Y$, $\alpha_{\mathbf{M I}}^{(\mathbf{n})}, \beta_{\mathbf{M I}}^{(\mathbf{n})}$, and $R_{\mathbf{M I}}^{(\mathbf{n})}$ as follows:

$$
\lambda_{\max }^{(\mathbf{n})}=Y \sqrt{\left\{\left(\alpha_{\mathbf{M I}}^{(\mathbf{n})}\right)^{2}+\left(\beta_{\mathbf{M I}}^{(\mathbf{n})}\right)^{2}\right\}}+R_{\mathbf{M I}}^{(\mathbf{n})} .
$$

If the FCA and FCD are neglected, the expression of maximum gain becomes

$$
\lambda_{\max }^{(\mathbf{n})}=Y \sqrt{\left(\frac{n-1}{n}\right)^{2} Q_{\mathbf{n}}^{2} Y^{2(n-2)}+1}-\left(1+Q_{\mathbf{n}} Y^{n-1}\right) .
$$

We further deduce the expression for the minimum intensity to initiate the MI. It can be shown after algebraic 


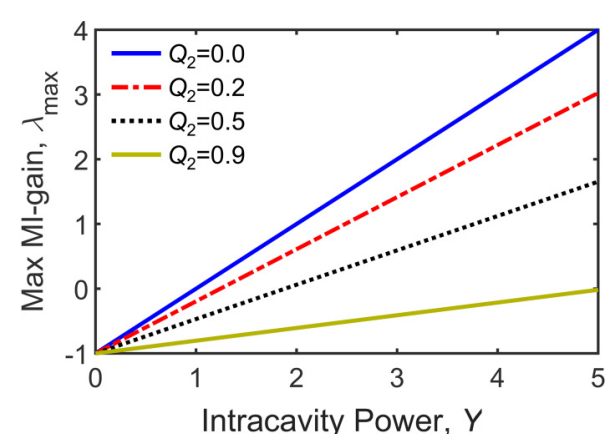

(a)

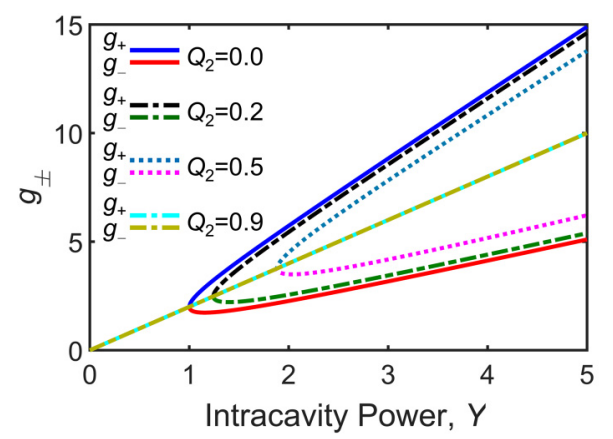

(b)

FIG. 10. (a) Maximum MI gain with the change in intracavity power $Y$ with a set of four 2PA coefficients $\left(Q_{2}=0,0.2,0.5,0.9\right)$. (b) Range of $\Delta$ for which MI can initiate with different 2PA coefficients $\left(Q_{2}=0,0.2,0.5,0.9\right)$.

simplification that the steady-state solution will always be stable for

$$
Y \leqslant \frac{-R_{\mathbf{M I}}^{(\mathbf{n})}}{\sqrt{\left\{\left(\alpha_{\mathbf{M I}}^{(\mathbf{n})}\right)^{2}+\left(\beta_{\mathbf{M I}}^{(\mathbf{n})}\right)^{2}\right\}}} .
$$

As an example, in the presence of only 2PA, the value of $Y$ for which the steady-state solution of the LLE will always be stable in the presence of only $2 \mathrm{PA}$ is

$$
Y \leqslant \frac{1}{\left(\sqrt{\left(\frac{Q_{2}^{2}}{4}+1\right)}-Q_{2}\right)} .
$$

Maximum MI gain $\lambda_{\max }$ is plotted in Fig. 10(a) for four different values $(0,0.2,0.5$, and 0.9$)$ of $2 \mathrm{PA}$ coefficients $Q_{2}$ in the absence of FCA-FCD. It should be noted that the 2PA coefficient for silicon at telecom wavelength $Q_{2}$ is $\sim 0.9$.

\section{RANGE OF NORMALIZED DETUNING TO OBTAIN MI}

In this section, we find the range of possible normalized detuning to initiate the MI and compare the results for different cases such as the following: when all the nonlinear losses are absent, when only nPA is present, when both the nPA and FCA are present, and when all the nonlinear losses are present. The condition for threshold can be found by equating $\lambda$ given by Eq. (49) to 0. It is known [54] that in the absence of nonlinear losses,

$$
\frac{s}{2} \Omega^{2}=\left(\Delta-g_{ \pm}\right)
$$

where $g_{ \pm}$can be expressed in terms of $Y$,

$$
g_{ \pm}=2 Y \pm \sqrt{Y^{2}-1}
$$

It is to be noted, in the anomalous dispersion region, for $\Omega$ to possess real solution, $\Delta$ must be less than $g_{+}$whereas, for the normal dispersion regime, the required detuning $\Delta$ should be more than $\mathrm{g}_{-}$for MI to occur. When the pump detuning lies in between $\left(g_{-}, g_{+}\right) \in$, i.e., $g_{-}<\Delta<\mathrm{g}_{+}$, MI can be initiated for both the anomalous and normal dispersion regimes. Similarly, in the presence of all the nonlinear losses, the general expression to obtain the threshold condition can be determined from Eq. (44):

$$
\left\{I_{\mathbf{M I}}^{(\mathbf{n})}\right\}^{2}=\left\{\left(\alpha_{\mathbf{M I}}^{(\mathbf{n})}\right)^{2}+\left(\beta_{\mathbf{M I}}^{(\mathbf{n})}\right)^{2}\right\} Y^{2}-\left\{R_{\mathbf{M I}}^{(\mathbf{n})}\right\}^{2}
$$

However, the relation of MI gain, $\Omega$, with $\Delta$ and output power $Y$ obtained from Eqs. (53)-(55) can be given by a thirdorder polynomial of $\Omega$,

$$
-\frac{d_{3}}{6} \Omega^{3}+\frac{s}{2} \Omega^{2}=\left(\Delta-g_{ \pm}^{(\mathbf{n})^{\prime}}\right),
$$

where

$$
\begin{gathered}
g_{1_{ \pm}}^{(\mathbf{n})}=2 Y \pm \sqrt{\left\{\left(\alpha_{\mathbf{M I}}^{(\mathbf{n})}\right)^{2}+\left(\beta_{\mathbf{M I}}^{(\mathbf{n})}\right)^{2}\right\} Y^{2}-\left(R_{\mathbf{M I}}^{(\mathbf{n})}\right)^{2}} \\
g_{ \pm}^{(\mathbf{n})^{\prime}}=g_{1_{ \pm}}^{(\mathbf{n})}-\frac{K C_{\mathbf{n}}}{2} Y^{n} .
\end{gathered}
$$

If the carrier lifetime can be reduced by sweeping the carriers applied to the external bias voltage across the device cross section, which often can be done by forming a $p-i-n$ junction across the waveguide cross section, then the square of the $\tau_{c}$ term can be negligible. The situation is equivalent to the case when nPA is present while FCA and FCD are absent. In this case, neglecting the higher-order dispersion terms $\left[d_{k}(k \geqslant 3)=0\right]$ Eqs. (56)-(58) are reduced to

$$
\frac{s}{2} \Omega^{2}=\left(\Delta-g_{1_{ \pm}}^{(\mathbf{n})}\right) \text {. }
$$

For 2PA without FCA-FCD,

$$
\frac{s}{2} \Omega^{2}=\left(\Delta-g_{1_{ \pm}}^{(2)}\right)
$$

$g_{1_{ \pm}}^{(2)}$

$$
=2 Y \pm \sqrt{\left[\left(\frac{n-1}{n}\right)^{2} Q_{\mathbf{n}}^{2} Y^{2(n-2)}+1\right] Y^{2}-\left(1+Q_{\mathbf{n}} Y^{n-1}\right)^{2}}
$$

In Fig. 10(b) we plot the range of $\Delta$ both in the absence of all nonlinear losses and in the presence of only 2PA, for which the steady-state solution can be unstable. It is discussed earlier that in the absence of all nonlinear losses the steady-state solution will be unstable in the range $g_{-}<\Delta<g_{+}$where $g_{ \pm}$ is given by Eq. (60). From Fig. 10(b) it can be seen clearly that with the gradual increase in the 2PA coefficient, the range of $\Delta$ for which MI can initiate becomes narrower. 
There is also an in-depth existing study on optical microcavity that discusses the generation of the Turing pattern analytically for two distinct cases considering either intensitydependent nonlinear losses or the intensity-dependent nonlinear refractive index of the medium. However, the mathematical framework outlined in [62] did not include the effects of multiphoton absorptions, FCA, and FCD. Another interesting work led by Chembo (Godey et al.) [63] presents the generation of Turing rolls as well as the generation of bright and dark temporal solitons in anomalous and normal dispersion regimes, respectively, without considering nonlinear losses. We believe that our work can further be extended following the mathematical analysis given in $[62,63]$ including all the nonlinear losses as our formalism is amenable to the incorporation of other associated physical effects like thermal detuning [61].

\section{CONCLUSIONS}

To conclude our work, we have derived analytical expressions of steady-state homogeneous solutions of a free-carrierdriven Kerr frequency comb. Higher-order $(>3)$ characteristic polynomials of intracavity power describing the steady-state homogeneous solution of the modified LLE are discussed in detail. The nonlinear phase detuning of the cavity has been observed through negative Kerr tilt. We also find an analytical expression for the steady-state FCD-induced cavity detuning. Expression of MI gain in the presence of all nonlinear losses is found and the threshold detuning along with the range of normalized pump detuning to initiate MI are discussed. The maximum allowed value of the 2PA coefficient for which the optical bistability can occur and the analytical expression of the threshold pump detuning to initiate the MI are important parameters from the experimental point of view. Therefore, our theoretical study is a step towards predicting comb dynamics in the realistic cases where all the nonlinear losses and higher-order dispersions are present.

\section{ACKNOWLEDGMENTS}

V.M. acknowledges financial support under the CSIR SRF-scheme from Council of Scientific and Industrial Research (CSIR), Government of India. The authors would also like to thank Dr. Samudra Roy, Assistant Professor, Department of Physics, Indian Institute of Technology, Kharagpur and Dr. Jae K. Jang, postdoctoral fellow, Columbia University, USA for their valuable discussions and suggestions related to the theoretical modeling of the frequency comb.
[1] S. T. Cundiff and J. Ye, Colloquium: Femtosecond optical frequency combs, Rev. Mod. Phys. 75, 325 (2003).

[2] T. Fortier, M. Kirchner, F. Quinlan, J. Taylor, J. Bergquist, T. Rosenband, N. Lemke, A. Ludlow, Y. Jiang, C. Oates, and S. Diddams, Generation of ultrastable microwaves via optical frequency division, Nat. Photonics 5, 425 (2011).

[3] J. Wu, X. Xu, T. G. Nguyen, S. T. Chu, R. Morandotti, A. Mitchell, and D. J. Moss, Harnessing optical micro-combs for microwave photonics, IEEE J. Sel. Top. Quantum Electron. 24, 6101020 (2018).

[4] Y. Chembo, Kerr optical frequency combs: theory, applications, and perspectives, Nanophotonics 5, 214 (2016).

[5] J. Pfeifle, V. Brasch, M. Lauermann, Y. Yu, D. Wegner, T. Herr, K. Hartinger, P. Schindler, J. Li, D. Hillerkuss, R. Schmogrow, C. Weimann, R. Holzwarth, W. Freude, J. Leuthold, T. Kippenberg, and C. Koos, Coherent terabit communications with microresonator Kerr frequency combs, Nat. Photonics 8, 375 (2014).

[6] S. A. Diddams, L. Hollberg, and V. Mbele, Molecular fingerprinting with the resolved modes of a femtosecond laser frequency comb, Nature 445, 627 (2007).

[7] T. Steinmetz, T. Wilken, C. Araujo-Hauck, R. Holzwarth, T. Hansch, L. Pasquini, A. Manescau, S. D'Odorico, M. Murphy, T. Kentischer, W. Schmidt, and T. Udem, Laser frequency combs for astronomical observations, Science 321, 1335 (2008).

[8] Y. Chembo, Quantum dynamics of Kerr optical frequency combs below and above threshold: Spontaneous four-wave mixing, entanglement, and squeezed states of light, Phys. Rev. A 93, 033820 (2016).

[9] T. Udem, J. Reichert, R. Holzwarth, and T. W. Hänsch, Absolute Optical Frequency Measurement of the Cesium $D_{1}$ Line with a Mode-Locked Laser, Phys. Rev. Lett. 82, 3568 (1999).
[10] T. Kippenberg, R. Holzwarth, and S. Diddams, Microresonatorbased optical frequency combs, Science 332, 555 (2011).

[11] P. Del'Haye, A. Schliesser, O. Arcizet, T. Wilken, R. Holzwarth, and T. Kippenberg, Optical frequency comb generation from a monolithic microresonator, Nature 450, 1214 (2007).

[12] T. Herr, K. Hartinger, J. Riemensberger, C. Wang, E. Gavartin, R. Holzwarth, M. Gorodetsky, and T. Kippenberg, Universal formation dynamics and noise of Kerr-frequency combs in microresonators, Nat. Photonics 6, 480 (2012).

[13] T. Kippenberg, S. Spillane, and K. Vahala, Kerr-Nonlinearity Optical Parametric Oscillation in an Ultrahigh- $Q$ Toroid Microcavity, Phys. Rev. Lett. 93, 083904 (2004).

[14] A. Griffith, J. Cardenas, C. B. Poitras, and M. Lipson, Highquality factor and high confinement silicon resonators using etchless process, Opt. Express 20, 21341 (2012).

[15] A. Savchenkov, A. Matsko, V. Ilchenko, I. Solomatine, D. Seidel and L. Maleki, Tunable Optical Frequency Comb with a Crystalline Whispering Gallery Mode Resonator, Phys. Rev. Lett. 101, 093902 (2008).

[16] R. Henriet, G. Lin, A. Coillet, M. Jacquot, L. Furfaro, L. Larger, and Y. K. Chembo, Kerr optical frequency comb generation in strontium fluoride whispering-gallery-mode resonators with billion quality factor, Opt. Lett. 40, 1567 (2015).

[17] W. Liang, A. A. Savchenkov, A. B. Matsko, V. S. Ilchenko, D. Seidel, and L. Maleki, Generation of near-infrared frequency combs from a $\mathrm{MgF}_{2}$ whispering gallery mode resonator, Opt. Lett. 36, 2290 (2011).

[18] G. Lin, S. Diallo, R. Henriet, M. Jacquot, and Y. K. Chembo, Barium fluoride whispering-gallery-mode disk-resonator with one billion quality-factor, Opt. Lett. 39, 6009 (2014).

[19] C. Wang, T. Herr, P. Del'Haye, A. Schliesser, J. Hofer, R. Holzwarth, T. Hänsch, N. Picqué, and T. Kippenberg, 
Mid-infrared optical frequency combs at $2.5 \mu \mathrm{m}$ based on crystalline microresonators, Nat. Commun. 4, 1345 (2013).

[20] L. Razzari, D. Duchesne, M. Ferrera, R. Morandotti, S. Chu, B. Little, and D. Moss, CMOS-compatible integrated optical hyper-parametric oscillator, Nat. Photonics 4, 41 (2000).

[21] V. Venkataraman, B. Hausmann, M. Loncar, I. Bulu, and P. Deotare, Diamond: a new platform for nonlinear photonics, SPIE Newsroom (2014), doi: 10.1117/2.1201406.005520.

[22] S. Papp and S. Diddams, Spectral and temporal characterization of a fused-quartz-microresonator optical frequency comb, Phys. Rev. A 84, 053833 (2011).

[23] H. Jung, C. Xiong, K. Fong, X. Zhang, and H. Tang, Optical frequency comb generation from aluminum nitride microring resonator, Opt. Lett. 38, 2810 (2013).

[24] H. Jung and H. Tang, Aluminum nitride as nonlinear optical material for on-chip frequency comb generation and frequency conversion, Nanophotonics 5, 263 (2016).

[25] M. Zhang, C. Wang, R. Cheng, A. Shams-Ansari, and M. Lončar, Monolithic ultra-high- $Q$ lithium niobate microring resonator, Optica 4, 1536 (2017).

[26] M. Pu, L. Ottaviano, E. Semenova, and K. Yvind, Efficient frequency comb generation in AlGaAs-on-insulator, Optica 3, 823 (2016).

[27] K. Saha, Y. Okawachi, B. Shim, J. S. Levy, R. Salem, A. R. Johnson, M. A. Foster, M. R. E. Lamont, M. Lipson, and A. L. Gaeta, Modelocking and femtosecond pulse generation in chipbased frequency combs, Opt. Express 21, 1335 (2013).

[28] X. Shen, R. Beltran, V. Diep, S. Soltani, and A. Armani, Low-threshold parametric oscillation in organically modified microcavities, Sci, Adv. 4, eaao4507 (2018).

[29] L. Pavesi and D. J. Lockwood, Silicon Photonics (Springer, Berlin, 2004).

[30] Q. Xu, D. Fattal, and R. Beausoleil, Silicon microring resonators with $1.5 \mu \mathrm{m}$ radius, Opt. Express 16, 4309 (2008).

[31] Q. Lin, O. J. Painter, and G. P. Agrawal, Nonlinear optical phenomena in silicon waveguides: Modeling and applications, Opt. Express 15, 16604 (2007).

[32] J. Leuthold, C. Koos, and W. Freude, Nonlinear silicon photonics, Nat. Photonics 4, 535 (2010).

[33] L. Zhang, Y. Yue, R. G. Beausoleil, and A. E. Willner, Analysis and engineering of chromatic dispersion in silicon waveguide bends and ring resonators, Opt. Express 19, 8102 (2011).

[34] B. Jalali, Nonlinear optics in the mid-infrared, Nat. Photonics 4, 506 (2010).

[35] L. Yin, Q. Lin, and G. P. Agrawal, Soliton fission and supercontinuum generation in silicon waveguides, Opt. Lett. 32, 391 (2007).

[36] Q. Xu and M. Lipson, All-optical logic based on silicon microring resonators, Opt. Express 15, 924 (2007).

[37] C. Koos, L. Jacome, C. Poulton, J. Leuthold, and W. Freude, Nonlinear silicon-on-insulator waveguides for all-optical signal processing, Opt. Express 15, 5976 (2007).

[38] K. Li and A. C. Foster, Nonlinear optics in hydrogenated amorphous silicon, IEEE J. Sel. Top. Quantum Electron. 24, 8201212 (2018).

[39] C. Husko, A. Clark, M. Collins, A. De Rossi, S. Combrié, G. Lehoucq, I. Rey, T. Krauss, C. Xiong, and B. Eggleton, Multiphoton absorption limits to heralded single photon sources, Sci. Rep. 3, 3087 (2013).
[40] R. Sun, P. Dong, N. Feng, C. Hong, J. Michel, M. Lipson, and L. Kimerling, Horizontal single and multiple slot waveguides: optical transmission at $\lambda=1550 \mathrm{~nm}$, Opt. Express 15, 17967 (2007).

[41] R. K. W. Lau, M. R. E. Lamont, Y. Okawachi, and A. L. Gaeta, Effects of multiphoton absorption on parametric comb generation in silicon microresonators, Opt. Lett. 40, 2778 (2015).

[42] A. Dutt, K. Luke, S. Manipatruni, A. Gaeta, P. Nussenzveig, and M. Lipson, On-Chip Optical Squeezing, Phys. Rev. Appl. 3, 044005 (2015).

[43] Y. Okawachi, K. Saha, J. S. Levy, Y. Henry Wen, M. Lipson, and A. L. Gaeta, Octave-spanning frequency comb generation in a silicon nitride chip, Opt. Lett. 36, 3398 (2011).

[44] A. Griffith, R. Lau, J. Cardenas, Y. Okawachi, A. Mohanty, R. Fain, Y. Lee, M. Yu, C. Phare, C. Poitras, A. Gaeta, and M. Lipson, Silicon-chip mid-infrared frequency comb generation, Nat. Commun. 6, 6299 (2015).

[45] I. Demirtzioglou, C. Lacava, K. R. H. Bottrill, D. J. Thomson, G. T. Reed, D. J. Richardson, and P. Petropoulos, Frequency comb generation in a silicon ring resonator modulator, Opt. Express 26, 790 (2018).

[46] S. Coen, H. G. Randle, T. Sylvestre, and M. Erkintalo, Modeling of octave-spanning Kerr frequency combs using a generalized mean-field Lugiato-Lefever model, Opt. Lett. 38, 37 (2013).

[47] T. Hansson, D. Modotto, and S. Wabnitz, Mid-infrared soliton and Raman frequency comb generation in silicon microrings, Opt. Lett. 39, 6747 (2014).

[48] M. Yu, Y. Okawachi, A. G. Griffith, M. Lipson, and A. L. Gaeta, Mode-locked mid-infrared frequency combs in a silicon microresonator, Optica 3, 854 (2016).

[49] M. Yu, J. Jang, Y. Okawachi, A. Griffith, K. Luke, S. Miller, X. Ji, M. Lipson, and A. Gaeta, Breather soliton dynamics in microresonators, Nat. Commun. 8, 14569 (2017).

[50] S. Malaguti, G. Bellanca, A. de. Rossi, S. Combrié, and S. Trillo, Self-pulsing driven by two-photon absorption in semiconductor nanocavities, Phys. Rev. A. 83, 051802(R) (2011).

[51] R. Haldar, P. Mondal, V. Mishra, and S. K. Varshney, Stability analysis and bandwidth estimation of free-carrier driven Kerr frequency-combs, in 2017 Conference on Lasers and ElectroOptics Pacific Rim (CLEO-PR) (IEEE, Singapore, 2017).

[52] S. Coen and M. Erkintalo, Universal scaling laws of Kerr frequency combs, Opt. Lett. 38, 1790 (2013).

[53] M. Haelterman, S. Trillo, and S. Wabnitz, Additive-modulationinstability ring laser in the normal dispersion regime of a fiber, Opt. Lett. 17, 745 (1992).

[54] T. Hansson, D. Modotto, and S. Wabnitz, Dynamics of the modulational instability in microresonator frequency combs, Phys. Rev. A 88, 023819 (2013).

[55] Y. Chembo and N. Yu, Modal expansion approach to opticalfrequency-comb generation with monolithic whisperinggallery-mode resonators, Phys. Rev. A. 82, 033801 (2010).

[56] R. Haldar, A. Roy, P. Mondal, V. Mishra, and S. K. Varshney, Generalized mathematical formalism governing freecarrier driven Kerr frequency comb in optical micro-cavities, arXiv:1802.00391.

[57] Y. Okawachi, M. Yu, K. Luke, D. O. Carvalho, S. Ramelow, A. Farsi, M. Lipson, and A. L. Gaeta, Dual-pumped degenerate Kerr oscillator in a silicon nitride microresonator, Opt. Lett. 40, 5267 (2015). 
[58] Nonlinear Optical Cavity Dynamics: From Microresonators to Fiber Lasers, edited by P. Grelu (Wiley, New York, 2016).

[59] A. Sahoo and S. Roy, Effect of two-photon absorption on cavity soliton: stability and perturbation analysis, in Proceedings of Frontiers in Optics 2017, OSA Technical Digest (Optical Society of America, Washington, DC, 2017), paper JW4A.118.

[60] R. Hamerly, D. Gray, C. Rogers, and K. Jamshidi, Conditions for parametric and free-carrier oscillations in silicon ring cavities, J. Lightwave Technol. 36, 4671 (2018).
[61] C. Bao, Y. Xuan, J. A. Jaramillo-Villegas, D. E. Leaird, M. Qi, and A. M. Weiner, Direct soliton generation in microresonators, Opt. Lett. 42, 2519 (2017).

[62] G. Kozyreff, Localized Turing patterns in nonlinear optical cavities, Phys. D (Amsterdam, Neth.) 241, 939 (2012).

[63] C. Godey, I. V. Balakireva, A. Coillet, and Y. K. Chembo, Stability analysis of the spatiotemporal Lugiato-Lefever model for optical frequency combs in the anomalous and normal dispersion regimes, Phys. Rev. A 89, 063814 (2014). 